Acta Cryst. (2003). C59, 000-000

\title{
Crystal and Molecular Structure of $\left\{\left(\eta^{3}-I n d\right) \operatorname{Pd}(\mu-C l)\right\}_{2}$
}

Gary D. Enright ${ }^{a}{ }^{\text {Davit }}$ Zargarian ${ }^{b}$ And Christine Sui-Seng ${ }^{c}$

${ }^{a}$ Steacie Institute for Molecular Science, National Research Council, Ottawa, Ontario, Canada, K1A 0R6, ${ }^{b}$ Département de Chimie, Université de Montréal, C.P. 6128, Succ. Centre-ville, Montréal, Québec, Canada, H3C 3J\%, and ${ }^{c}$ Département de Chimie, Université de Montréal, C.P. 6128, Succ. Centre-ville, Montréal, Québec, Canada H3C 3J\%. E-mail: zargarian. davit@umontreal.ca

\section{Abstract}

\section{Comment}

\section{Experimental}

Crystal data

$\mathrm{C}_{18} \mathrm{H}_{14} \mathrm{Cl}_{2} \mathrm{Pd}_{2}$

$M_{r}=513.99$

Triclinic

$P \overline{1}$

$a=10.2103$ (6) §

$b=12.5513(7) \AA$

$c=12.7249$ (7) $\AA$

$\alpha=87.9700(10)^{\circ}$

$\beta=78.2090(10)^{\circ}$

$\gamma=89.4970(10)^{\circ}$

$V=1595.32(16) \AA^{3}$

$Z=4$

$D_{x}=2.140 \mathrm{Mg} \mathrm{m}^{-3}$

$D_{m}$ not measured

Mo $K \alpha$ radiation

$\lambda=0.71073 \AA$
Cell parameters from 7834 reflections

$\theta=2.83-72.60^{\circ}$

$\mu=2.580 \mathrm{~mm}^{-1}$

$T=173(2) \mathrm{K}$

Block

Orange

$0.50 \times 0.25 \times 0.15 \mathrm{~mm}$

Crystal source: synthesized by the authors.

See text 
Data collection

Bruker AXS Smart 1K/Platform diffractometer

$\omega$ scans

Absorption correction:

multi-scan Sadabs (Sheldrick,1996)

$T_{\min }=0.466, T_{\max }=0.679$

27417 measured reflections

9004 independent reflections

Refinement

Refinement on $F^{2}$

$R\left[F^{2}>2 \sigma\left(F^{2}\right)\right]=0.0596$

$w R\left(F^{2}\right)=0.1688$

$S=0.894$

20303 reflections

398 parameters

H-atom parameters constrained
11089 reflections with

$$
>2 \operatorname{sigma}(I)
$$

$R_{\text {int }}=0.041$

$\theta_{\max }=29.62^{\circ}$

$h=-14 \rightarrow 14$

$k=-17 \rightarrow 17$

$l=-17 \rightarrow 17$

227 standard reflections

every ? reflections

intensity decay: $0.35 \%$

$$
\begin{aligned}
& w=1 /\left[\sigma^{2}\left(F_{o}^{2}\right)+(0.0943 P)^{2}+0.0000 P\right] \\
& \text { where } P=\left(F_{o}^{2}+2 F_{c}^{2}\right) / 3 \\
& (\Delta / \sigma)_{\max }=0.001 \\
& \Delta \rho_{\max }=2.854{\mathrm{e} \AA^{-3}}^{-3} \rho_{\min }=-2.471 \mathrm{e} \AA^{-3}
\end{aligned}
$$

Extinction correction: none

Scattering factors from International Tables for Crystallography (Vol. C) 
Table 1. Selected geometric parameters $\left(\AA,^{\circ}\right)$

\begin{tabular}{|c|c|c|c|}
\hline $\mathrm{Pd} 1-\mathrm{C} 12$ & $2.127(7)$ & $\mathrm{Pd} 3-\mathrm{C} 31$ & $2.208(6)$ \\
\hline $\mathrm{Pd} 1-\mathrm{C} 13$ & $2.172(7)$ & $\mathrm{Pd} 3-\mathrm{Cl} 3^{\mathrm{iii}}$ & $2.3962(16)$ \\
\hline $\mathrm{Pd} 1-\mathrm{C} 11$ & $2.194(6)$ & $\mathrm{Pd} 3-\mathrm{Cl} 3$ & $2.4086(17)$ \\
\hline $\mathrm{Pd} 1-\mathrm{Cl} 1$ & $2.3950(16)$ & $\mathrm{Pd} 3-\mathrm{C} 33 \mathrm{a}$ & $2.631(6)$ \\
\hline $\mathrm{Pd} 1-\mathrm{Cl}^{\mathrm{i}}$ & $2.4035(17)$ & $\mathrm{Cl} 3-\mathrm{Pd} 3^{\mathrm{iii}}$ & $2.3962(16)$ \\
\hline $\mathrm{Cl} 1-\mathrm{Pd} 1^{\mathrm{i}}$ & $2.4035(17)$ & $\mathrm{C} 31-\mathrm{C} 32$ & $1.421(9)$ \\
\hline $\mathrm{C} 11-\mathrm{C} 12$ & $1.447(10)$ & $\mathrm{C} 31-\mathrm{C} 37 \mathrm{a}$ & $1.485(10)$ \\
\hline $\mathrm{C} 11-\mathrm{C} 17 \mathrm{a}$ & $1.493(9)$ & $\mathrm{C} 32-\mathrm{C} 33$ & $1.389(10)$ \\
\hline $\mathrm{C} 12-\mathrm{C} 13$ & $1.385(10)$ & $\mathrm{C} 33-\mathrm{C} 33 \mathrm{a}$ & $1.476(10)$ \\
\hline $\mathrm{C} 13-\mathrm{C} 13 \mathrm{a}$ & $1.480(9)$ & $\mathrm{C} 33 \mathrm{a}-\mathrm{C} 34$ & $1.351(10)$ \\
\hline $\mathrm{C} 13 \mathrm{a}-\mathrm{C} 14$ & $1.365(9)$ & $\mathrm{C} 33 \mathrm{a}-\mathrm{C} 37 \mathrm{a}$ & $1.418(9)$ \\
\hline $\mathrm{C} 13 \mathrm{a}-\mathrm{C} 17 \mathrm{a}$ & $1.423(9)$ & $\mathrm{C} 34-\mathrm{C} 35$ & $1.397(12)$ \\
\hline $\mathrm{C} 14-\mathrm{C} 15$ & $1.375(10)$ & $\mathrm{C} 35-\mathrm{C} 36$ & $1.367(13)$ \\
\hline $\mathrm{C} 15-\mathrm{C} 16$ & $1.405(10)$ & $\mathrm{C} 36-\mathrm{C} 37$ & $1.397(11)$ \\
\hline $\mathrm{C} 16-\mathrm{C} 17$ & $1.386(9)$ & $\mathrm{C} 37-\mathrm{C} 37 \mathrm{a}$ & $1.383(10)$ \\
\hline $\mathrm{C} 17-\mathrm{C} 17 \mathrm{a}$ & $1.376(9)$ & $\mathrm{Pd}-\mathrm{C} 2$ & $2.148(7)$ \\
\hline $\mathrm{Pd} 2-\mathrm{C} 22$ & $2.130(7)$ & $\mathrm{Pd}-\mathrm{C} 1$ & $2.158(7)$ \\
\hline $\mathrm{Pd} 2-\mathrm{C} 21$ & $2.176(7)$ & $\mathrm{Pd}-\mathrm{C} 3$ & $2.202(6)$ \\
\hline $\mathrm{Pd} 2-\mathrm{C} 23$ & $2.213(7)$ & $\mathrm{Pd}-\mathrm{Cl}^{\mathrm{iv}}$ & $2.3913(16)$ \\
\hline $\mathrm{Pd} 2-\mathrm{Cl} 2$ & $2.3969(17)$ & $\mathrm{Pd}-\mathrm{Cl}$ & $2.4122(16)$ \\
\hline $\mathrm{Pd} 2-\mathrm{Cl} 2^{\mathrm{ii}}$ & $2.4040(17)$ & $\mathrm{Pd}-\mathrm{C} 7 \mathrm{a}$ & $2.625(6)$ \\
\hline $\mathrm{Cl} 2-\mathrm{Pd} 2^{\mathrm{ii}}$ & $2.4040(17)$ & $\mathrm{Pd}-\mathrm{C} 3 \mathrm{a}$ & $2.645(6)$ \\
\hline $\mathrm{C} 21-\mathrm{C} 22$ & $1.427(9)$ & $\mathrm{Cl}-\mathrm{Pd}^{\mathrm{iv}}$ & $2.3913(16)$ \\
\hline $\mathrm{C} 21-\mathrm{C} 27 \mathrm{a}$ & $1.509(9)$ & $\mathrm{C} 1-\mathrm{C} 2$ & $1.416(10)$ \\
\hline $\mathrm{C} 22-\mathrm{C} 23$ & $1.414(10)$ & $\mathrm{C} 1-\mathrm{C} 7 \mathrm{a}$ & $1.501(9)$ \\
\hline $\mathrm{C} 23-\mathrm{C} 23 \mathrm{a}$ & $1.468(8)$ & $\mathrm{C} 2-\mathrm{C} 3$ & $1.414(9)$ \\
\hline $\mathrm{C} 23 \mathrm{a}-\mathrm{C} 24$ & $1.391(9)$ & $\mathrm{C} 3-\mathrm{C} 3 \mathrm{a}$ & $1.471(9)$ \\
\hline $\mathrm{C} 23 \mathrm{a}-\mathrm{C} 27 \mathrm{a}$ & $1.400(9)$ & $\mathrm{C} 3 \mathrm{a}-\mathrm{C} 4$ & $1.390(8)$ \\
\hline $\mathrm{C} 24-\mathrm{C} 25$ & $1.401(9)$ & $\mathrm{C} 3 \mathrm{a}-\mathrm{C} 7 \mathrm{a}$ & $1.436(9)$ \\
\hline $\mathrm{C} 25-\mathrm{C} 26$ & $1.394(9)$ & $\mathrm{C} 4-\mathrm{C} 5$ & $1.395(11)$ \\
\hline $\mathrm{C} 26-\mathrm{C} 27$ & $1.374(10)$ & $\mathrm{C} 5-\mathrm{C} 6$ & $1.396(11)$ \\
\hline $\mathrm{C} 27-\mathrm{C} 27 \mathrm{a}$ & $1.380(9)$ & $\mathrm{C} 6-\mathrm{C} 7$ & $1.393(10)$ \\
\hline $\mathrm{Pd} 3-\mathrm{C} 32$ & $2.142(6)$ & $\mathrm{C} 7-\mathrm{C} 7 \mathrm{a}$ & $1.361(10)$ \\
\hline $\mathrm{Pd} 3-\mathrm{C} 33$ & $2.154(7)$ & & \\
\hline $\mathrm{C} 12-\mathrm{PD} 1-\mathrm{C} 13$ & $37.6(3)$ & C13-PD1-CL1 & $105.75(18)$ \\
\hline $\mathrm{C} 12-\mathrm{PD} 1-\mathrm{C} 11$ & $39.1(3)$ & C11-PD1-CL1 & $165.54(18)$ \\
\hline $\mathrm{C} 13-\mathrm{PD} 1-\mathrm{C} 11$ & $62.4(3)$ & $\mathrm{C} 12-\mathrm{PD} 1-\mathrm{CL} 1^{\mathrm{i}}$ & $132.9(2)$ \\
\hline $\mathrm{C} 12-\mathrm{PD} 1-\mathrm{CL} 1$ & $135.6(2)$ & $\mathrm{C} 13-\mathrm{PD} 1-\mathrm{CL} 1^{\mathrm{i}}$ & $165.89(18)$ \\
\hline
\end{tabular}




\begin{tabular}{|c|c|c|c|}
\hline $\mathrm{C} 11-\mathrm{PD} 1-\mathrm{CL} 1^{\mathrm{i}}$ & $103.80(19)$ & $\mathrm{C} 22-\mathrm{C} 23-\mathrm{C} 23 \mathrm{~A}$ & $108.0(6)$ \\
\hline $\mathrm{CL} 1-\mathrm{PD} 1-\mathrm{CL}^{\mathrm{i}}$ & $87.36(6)$ & $\mathrm{C} 22-\mathrm{C} 23-\mathrm{PD} 2$ & $67.8(4)$ \\
\hline $\mathrm{PD} 1-\mathrm{CL} 1-\mathrm{PD} 1^{\mathrm{i}}$ & $92.64(6)$ & $\mathrm{C} 23 \mathrm{~A}-\mathrm{C} 23-\mathrm{PD} 2$ & $90.9(4)$ \\
\hline $\mathrm{C} 12-\mathrm{C} 11-\mathrm{C} 17 \mathrm{~A}$ & $108.0(6)$ & $\mathrm{C} 24-\mathrm{C} 23 \mathrm{~A}-\mathrm{C} 27 \mathrm{~A}$ & $120.7(6)$ \\
\hline $\mathrm{C} 12-\mathrm{C} 11-\mathrm{PD} 1$ & $68.0(4)$ & $\mathrm{C} 24-\mathrm{C} 23 \mathrm{~A}-\mathrm{C} 23$ & $131.8(6)$ \\
\hline $\mathrm{C} 17 \mathrm{~A}-\mathrm{C} 11-\mathrm{PD} 1$ & $89.9(4)$ & $\mathrm{C} 27 \mathrm{~A}-\mathrm{C} 23 \mathrm{~A}-\mathrm{C} 23$ & $107.5(6)$ \\
\hline $\mathrm{C} 13-\mathrm{C} 12-\mathrm{C} 11$ & $106.1(6)$ & $\mathrm{C} 23 \mathrm{~A}-\mathrm{C} 24-\mathrm{C} 25$ & $118.0(6)$ \\
\hline $\mathrm{C} 13-\mathrm{C} 12-\mathrm{PD} 1$ & $73.0(4)$ & $\mathrm{C} 26-\mathrm{C} 25-\mathrm{C} 24$ & $120.4(6)$ \\
\hline $\mathrm{C} 11-\mathrm{C} 12-\mathrm{PD} 1$ & $72.9(4)$ & $\mathrm{C} 27-\mathrm{C} 26-\mathrm{C} 25$ & $121.3(6)$ \\
\hline $\mathrm{C} 12-\mathrm{C} 13-\mathrm{C} 13 \mathrm{~A}$ & $110.2(6)$ & $\mathrm{C} 26-\mathrm{C} 27-\mathrm{C} 27 \mathrm{~A}$ & $118.8(7)$ \\
\hline $\mathrm{C} 12-\mathrm{C} 13-\mathrm{PD} 1$ & $69.5(4)$ & $\mathrm{C} 27-\mathrm{C} 27 \mathrm{~A}-\mathrm{C} 23 \mathrm{~A}$ & $120.9(6)$ \\
\hline $\mathrm{C} 13 \mathrm{~A}-\mathrm{C} 13-\mathrm{PD} 1$ & $91.2(4)$ & $\mathrm{C} 27-\mathrm{C} 27 \mathrm{~A}-\mathrm{C} 21$ & $131.6(7)$ \\
\hline $\mathrm{C} 14-\mathrm{C} 13 \mathrm{~A}-\mathrm{C} 17 \mathrm{~A}$ & $119.5(6)$ & $\mathrm{C} 23 \mathrm{~A}-\mathrm{C} 27 \mathrm{~A}-\mathrm{C} 21$ & $107.5(6)$ \\
\hline $\mathrm{C} 14-\mathrm{C} 13 \mathrm{~A}-\mathrm{C} 13$ & $133.9(6)$ & $\mathrm{C} 32-\mathrm{PD} 3-\mathrm{C} 33$ & $37.7(3)$ \\
\hline $\mathrm{C} 17 \mathrm{~A}-\mathrm{C} 13 \mathrm{~A}-\mathrm{C} 13$ & $106.5(6)$ & $\mathrm{C} 32-\mathrm{PD} 3-\mathrm{C} 31$ & $38.1(2)$ \\
\hline $\mathrm{C} 13 \mathrm{~A}-\mathrm{C} 14-\mathrm{C} 15$ & $119.4(6)$ & $\mathrm{C} 33-\mathrm{PD} 3-\mathrm{C} 31$ & $61.7(3)$ \\
\hline $\mathrm{C} 14-\mathrm{C} 15-\mathrm{C} 16$ & $121.2(7)$ & $\mathrm{C} 32-\mathrm{PD} 3-\mathrm{CL} 3^{\mathrm{iii}}$ & $131.6(2)$ \\
\hline $\mathrm{C} 17-\mathrm{C} 16-\mathrm{C} 15$ & $120.1(7)$ & $\mathrm{C} 33-\mathrm{PD} 3-\mathrm{CL} 3^{\mathrm{iii}}$ & $102.7(2)$ \\
\hline $\mathrm{C} 17 \mathrm{~A}-\mathrm{C} 17-\mathrm{C} 16$ & $118.2(6)$ & $\mathrm{C} 31-\mathrm{PD} 3-\mathrm{CL} 3^{\mathrm{iii}}$ & $163.63(19)$ \\
\hline $\mathrm{C} 17-\mathrm{C} 17 \mathrm{~A}-\mathrm{C} 13 \mathrm{~A}$ & $121.5(6)$ & C32-PD3-CL3 & $138.0(2)$ \\
\hline $\mathrm{C} 17-\mathrm{C} 17 \mathrm{~A}-\mathrm{C} 11$ & $132.2(6)$ & C33-PD3-CL3 & $167.2(2)$ \\
\hline $\mathrm{C} 13 \mathrm{~A}-\mathrm{C} 17 \mathrm{~A}-\mathrm{C} 11$ & $106.4(6)$ & C31-PD3-CL3 & $107.95(19)$ \\
\hline $\mathrm{C} 22-\mathrm{PD} 2-\mathrm{C} 21$ & $38.7(3)$ & CL3 ${ }^{\mathrm{iii}}-\mathrm{PD} 3-\mathrm{CL} 3$ & $86.77(6)$ \\
\hline $\mathrm{C} 22-\mathrm{PD} 2-\mathrm{C} 23$ & $37.9(3)$ & $\mathrm{C} 32-\mathrm{PD} 3-\mathrm{C} 33 \mathrm{~A}$ & $57.9(2)$ \\
\hline $\mathrm{C} 21-\mathrm{PD} 2-\mathrm{C} 23$ & $63.1(3)$ & $\mathrm{C} 33-\mathrm{PD} 3-\mathrm{C} 33 \mathrm{~A}$ & $34.1(2)$ \\
\hline $\mathrm{C} 22-\mathrm{PD} 2-\mathrm{CL} 2$ & $138.4(2)$ & $\mathrm{C} 31-\mathrm{PD} 3-\mathrm{C} 33 \mathrm{~A}$ & $56.4(2)$ \\
\hline $\mathrm{C} 21-\mathrm{PD} 2-\mathrm{CL} 2$ & $166.54(19)$ & $\mathrm{CL} 3^{\mathrm{iii}}-\mathrm{PD} 3-\mathrm{C} 33 \mathrm{~A}$ & $108.14(16)$ \\
\hline $\mathrm{C} 23-\mathrm{PD} 2-\mathrm{CL} 2$ & $107.34(19)$ & $\mathrm{CL} 3-\mathrm{PD} 3-\mathrm{C} 33 \mathrm{~A}$ & $134.75(17)$ \\
\hline $\mathrm{C} 22-\mathrm{PD} 2-\mathrm{CL} 2^{\mathrm{ii}}$ & $130.4(2)$ & PD3 ${ }^{\mathrm{iii}}-\mathrm{CL} 3-\mathrm{PD} 3$ & $93.23(6)$ \\
\hline $\mathrm{C} 21-\mathrm{PD} 2-\mathrm{CL} 2^{\mathrm{ii}}$ & $101.7(2)$ & $\mathrm{C} 32-\mathrm{C} 31-\mathrm{C} 37 \mathrm{~A}$ & $109.1(6)$ \\
\hline $\mathrm{C} 23-\mathrm{PD} 2-\mathrm{CL} 2^{\mathrm{ii}}$ & $164.65(19)$ & $\mathrm{C} 32-\mathrm{C} 31-\mathrm{PD} 3$ & $68.4(4)$ \\
\hline $\mathrm{CL} 2-\mathrm{PD} 2-\mathrm{CL} 2^{\mathrm{ii}}$ & $87.37(6)$ & $\mathrm{C} 37 \mathrm{~A}-\mathrm{C} 31-\mathrm{PD} 3$ & $89.8(4)$ \\
\hline $\mathrm{PD} 2-\mathrm{CL} 2-\mathrm{PD} 2^{\mathrm{ii}}$ & $92.63(6)$ & $\mathrm{C} 33-\mathrm{C} 32-\mathrm{C} 31$ & $105.6(6)$ \\
\hline $\mathrm{C} 22-\mathrm{C} 21-\mathrm{C} 27 \mathrm{~A}$ & $105.8(6)$ & $\mathrm{C} 33-\mathrm{C} 32-\mathrm{PD} 3$ & $71.6(4)$ \\
\hline $\mathrm{C} 22-\mathrm{C} 21-\mathrm{PD} 2$ & $68.9(4)$ & $\mathrm{C} 31-\mathrm{C} 32-\mathrm{PD} 3$ & $73.5(4)$ \\
\hline $\mathrm{C} 27 \mathrm{~A}-\mathrm{C} 21-\mathrm{PD} 2$ & $90.3(4)$ & $\mathrm{C} 32-\mathrm{C} 33-\mathrm{C} 33 \mathrm{~A}$ & $110.2(6)$ \\
\hline $\mathrm{C} 23-\mathrm{C} 22-\mathrm{C} 21$ & $107.8(6)$ & $\mathrm{C} 32-\mathrm{C} 33-\mathrm{PD} 3$ & $70.7(4)$ \\
\hline $\mathrm{C} 23-\mathrm{C} 22-\mathrm{PD} 2$ & $74.2(4)$ & $\mathrm{C} 33 \mathrm{~A}-\mathrm{C} 33-\mathrm{PD} 3$ & $90.9(4)$ \\
\hline $\mathrm{C} 21-\mathrm{C} 22-\mathrm{PD} 2$ & $72.4(4)$ & $\mathrm{C} 34-\mathrm{C} 33 \mathrm{~A}-\mathrm{C} 37 \mathrm{~A}$ & $120.1(8)$ \\
\hline
\end{tabular}




\begin{tabular}{|c|c|c|c|}
\hline $\mathrm{C} 34-\mathrm{C} 33 \mathrm{~A}-\mathrm{C} 33$ & $133.6(8)$ & $\mathrm{CL}^{\mathrm{iv}}-\mathrm{PD}-\mathrm{C} 3 \mathrm{~A}$ & $134.20(15)$ \\
\hline $\mathrm{C} 37 \mathrm{~A}-\mathrm{C} 33 \mathrm{~A}-\mathrm{C} 33$ & $106.3(6)$ & $\mathrm{CL}-\mathrm{PD}-\mathrm{C} 3 \mathrm{~A}$ & $110.72(14)$ \\
\hline $\mathrm{C} 34-\mathrm{C} 33 \mathrm{~A}-\mathrm{PD} 3$ & $133.2(5)$ & $\mathrm{C} 7 \mathrm{~A}-\mathrm{PD}-\mathrm{C} 3 \mathrm{~A}$ & $31.62(19)$ \\
\hline $\mathrm{C} 37 \mathrm{~A}-\mathrm{C} 33 \mathrm{~A}-\mathrm{PD} 3$ & $75.4(4)$ & $\mathrm{PD}^{\mathrm{iv}}-\mathrm{CL}-\mathrm{PD}$ & $93.26(5)$ \\
\hline $\mathrm{C} 33-\mathrm{C} 33 \mathrm{~A}-\mathrm{PD} 3$ & $55.0(3)$ & $\mathrm{C} 2-\mathrm{C} 1-\mathrm{C} 7 \mathrm{~A}$ & $108.9(6)$ \\
\hline $\mathrm{C} 33 \mathrm{~A}-\mathrm{C} 34-\mathrm{C} 35$ & $119.1(8)$ & $\mathrm{C} 2-\mathrm{C} 1-\mathrm{PD}$ & $70.4(4)$ \\
\hline $\mathrm{C} 36-\mathrm{C} 35-\mathrm{C} 34$ & $120.8(8)$ & $\mathrm{C} 7 \mathrm{~A}-\mathrm{C} 1-\mathrm{PD}$ & $89.9(4)$ \\
\hline $\mathrm{C} 35-\mathrm{C} 36-\mathrm{C} 37$ & $121.7(8)$ & $\mathrm{C} 3-\mathrm{C} 2-\mathrm{C} 1$ & $106.2(6)$ \\
\hline $\mathrm{C} 37 \mathrm{~A}-\mathrm{C} 37-\mathrm{C} 36$ & $116.8(8)$ & $\mathrm{C} 3-\mathrm{C} 2-\mathrm{PD}$ & $73.1(4)$ \\
\hline $\mathrm{C} 37-\mathrm{C} 37 \mathrm{~A}-\mathrm{C} 33 \mathrm{~A}$ & $121.4(7)$ & $\mathrm{C} 1-\mathrm{C} 2-\mathrm{PD}$ & $71.2(4)$ \\
\hline $\mathrm{C} 37-\mathrm{C} 37 \mathrm{~A}-\mathrm{C} 31$ & $132.7(7)$ & $\mathrm{C} 2-\mathrm{C} 3-\mathrm{C} 3 \mathrm{~A}$ & $109.2(6)$ \\
\hline $\mathrm{C} 33 \mathrm{~A}-\mathrm{C} 37 \mathrm{~A}-\mathrm{C} 31$ & $105.9(6)$ & $\mathrm{C} 2-\mathrm{C} 3-\mathrm{PD}$ & $69.0(4)$ \\
\hline $\mathrm{C} 2-\mathrm{PD}-\mathrm{C} 1$ & $38.4(3)$ & $\mathrm{C} 3 \mathrm{~A}-\mathrm{C} 3-\mathrm{PD}$ & $89.9(4)$ \\
\hline $\mathrm{C} 2-\mathrm{PD}-\mathrm{C} 3$ & $37.9(2)$ & $\mathrm{C} 4-\mathrm{C} 3 \mathrm{~A}-\mathrm{C} 7 \mathrm{~A}$ & $119.9(6)$ \\
\hline $\mathrm{C} 1-\mathrm{PD}-\mathrm{C} 3$ & $62.6(3)$ & $\mathrm{C} 4-\mathrm{C} 3 \mathrm{~A}-\mathrm{C} 3$ & $133.0(6)$ \\
\hline $\mathrm{C} 2-\mathrm{PD}-\mathrm{CL}^{\mathrm{iv}}$ & $132.20(19)$ & $\mathrm{C} 7 \mathrm{~A}-\mathrm{C} 3 \mathrm{~A}-\mathrm{C} 3$ & $107.0(5)$ \\
\hline $\mathrm{C} 1-\mathrm{PD}-\mathrm{CL}^{\mathrm{iv}}$ & $102.0(2)$ & $\mathrm{C} 4-\mathrm{C} 3 \mathrm{~A}-\mathrm{PD}$ & $134.5(5)$ \\
\hline $\mathrm{C} 3-\mathrm{PD}-\mathrm{CL}^{\mathrm{iv}}$ & $163.63(18)$ & $\mathrm{C} 7 \mathrm{~A}-\mathrm{C} 3 \mathrm{~A}-\mathrm{PD}$ & $73.4(4)$ \\
\hline $\mathrm{C} 2-\mathrm{PD}-\mathrm{CL}$ & $137.6(2)$ & $\mathrm{C} 3-\mathrm{C} 3 \mathrm{~A}-\mathrm{PD}$ & $56.3(3)$ \\
\hline $\mathrm{C} 1-\mathrm{PD}-\mathrm{CL}$ & $167.82(18)$ & $\mathrm{C} 3 \mathrm{~A}-\mathrm{C} 4-\mathrm{C} 5$ & $117.6(7)$ \\
\hline $\mathrm{C} 3-\mathrm{PD}-\mathrm{CL}$ & $107.72(17)$ & $\mathrm{C} 4-\mathrm{C} 5-\mathrm{C} 6$ & $121.8(7)$ \\
\hline $\mathrm{CL}^{\mathrm{iv}}-\mathrm{PD}-\mathrm{CL}$ & $86.74(5)$ & $\mathrm{C} 7-\mathrm{C} 6-\mathrm{C} 5$ & $120.8(7)$ \\
\hline $\mathrm{C} 2-\mathrm{PD}-\mathrm{C} 7 \mathrm{~A}$ & $58.6(2)$ & $\mathrm{C} 7 \mathrm{~A}-\mathrm{C} 7-\mathrm{C} 6$ & $118.3(7)$ \\
\hline $\mathrm{C} 1-\mathrm{PD}-\mathrm{C} 7 \mathrm{~A}$ & $34.9(2)$ & $\mathrm{C} 7-\mathrm{C} 7 \mathrm{~A}-\mathrm{C} 3 \mathrm{~A}$ & $121.6(6)$ \\
\hline $\mathrm{C} 3-\mathrm{PD}-\mathrm{C} 7 \mathrm{~A}$ & $57.1(2)$ & $\mathrm{C} 7-\mathrm{C} 7 \mathrm{~A}-\mathrm{C} 1$ & $133.0(7)$ \\
\hline $\mathrm{CL}^{\mathrm{iv}}-\mathrm{PD}-\mathrm{C} 7 \mathrm{~A}$ & $107.31(16)$ & $\mathrm{C} 3 \mathrm{~A}-\mathrm{C} 7 \mathrm{~A}-\mathrm{C} 1$ & $105.3(6)$ \\
\hline $\mathrm{CL}-\mathrm{PD}-\mathrm{C} 7 \mathrm{~A}$ & $134.45(15)$ & $\mathrm{C} 7-\mathrm{C} 7 \mathrm{~A}-\mathrm{PD}$ & $132.8(5)$ \\
\hline $\mathrm{C} 2-\mathrm{PD}-\mathrm{C} 3 \mathrm{~A}$ & $57.7(2)$ & $\mathrm{C} 3 \mathrm{~A}-\mathrm{C} 7 \mathrm{~A}-\mathrm{PD}$ & $74.9(4)$ \\
\hline $\mathrm{C} 1-\mathrm{PD}-\mathrm{C} 3 \mathrm{~A}$ & $57.1(2)$ & $\mathrm{C} 1-\mathrm{C} 7 \mathrm{~A}-\mathrm{PD}$ & $55.3(3)$ \\
\hline $\begin{array}{l}\mathrm{C} 3-\mathrm{PD}-\mathrm{C} 3 \mathrm{~A} \\
\mathrm{C} 12-\mathrm{PD} 1-\mathrm{CL} 1-\mathrm{PD} 1^{\mathrm{i}}\end{array}$ & $\begin{array}{r}33.8(2) \\
-158.0(3)\end{array}$ & $\mathrm{CL1} 1^{\mathrm{i}}-\mathrm{PD} 1-\mathrm{C} 11-\mathrm{C} 17 \mathrm{~A}$ & $106.4(4)$ \\
\hline $\mathrm{C} 13-\mathrm{PD} 1-\mathrm{CL} 1-\mathrm{PD} 1^{\mathrm{i}}$ & $174.71(19)$ & $\mathrm{C} 17 \mathrm{~A}-\mathrm{C} 11-\mathrm{C} 12-\mathrm{C} 13$ & $16.3(7)$ \\
\hline $\mathrm{C} 11-\mathrm{PD} 1-\mathrm{CL} 1-\mathrm{PD} 1^{\mathrm{i}}$ & $141.0(8)$ & $\mathrm{PD} 1-\mathrm{C} 11-\mathrm{C} 12-\mathrm{C} 13$ & $-66.0(5)$ \\
\hline $\mathrm{CL} 1^{\mathrm{i}}-\mathrm{PD} 1-\mathrm{CL} 1-\mathrm{PD} 1^{\mathrm{i}}$ & 0 & $\mathrm{C} 17 \mathrm{~A}-\mathrm{C} 11-\mathrm{C} 12-\mathrm{PD} 1$ & $82.3(4)$ \\
\hline $\mathrm{C} 13-\mathrm{PD} 1-\mathrm{C} 11-\mathrm{C} 12$ & $39.1(4)$ & $\mathrm{C} 11-\mathrm{PD} 1-\mathrm{C} 12-\mathrm{C} 13$ & $113.4(6)$ \\
\hline $\mathrm{CL} 1-\mathrm{PD} 1-\mathrm{C} 11-\mathrm{C} 12$ & $76.2(9)$ & $\mathrm{CL} 1-\mathrm{PD} 1-\mathrm{C} 12-\mathrm{C} 13$ & $-46.4(5)$ \\
\hline $\mathrm{CL} 1^{\mathrm{i}}-\mathrm{PD} 1-\mathrm{C} 11-\mathrm{C} 12$ & $-144.1(4)$ & $\mathrm{CL} 1{ }^{\mathrm{i}}-\mathrm{PD} 1-\mathrm{C} 12-\mathrm{C} 13$ & $164.4(3)$ \\
\hline $\mathrm{C} 12-\mathrm{PD} 1-\mathrm{C} 11-\mathrm{C} 17 \mathrm{~A}$ & $-109.5(6)$ & $\mathrm{C} 13-\mathrm{PD} 1-\mathrm{C} 12-\mathrm{C} 11$ & $-113.4(6)$ \\
\hline $\mathrm{C} 13-\mathrm{PD} 1-\mathrm{C} 11-\mathrm{C} 17 \mathrm{~A}$ & $-70.4(4)$ & $\mathrm{CL} 1-\mathrm{PD} 1-\mathrm{C} 12-\mathrm{C} 11$ & $-159.7(3)$ \\
\hline
\end{tabular}




\begin{tabular}{|c|c|c|c|}
\hline $\mathrm{CL} 1{ }^{\mathrm{i}}-\mathrm{PD} 1-\mathrm{C} 12-\mathrm{C} 11$ & $51.0(5)$ & $\mathrm{C} 21-\mathrm{PD} 2-\mathrm{C} 22-\mathrm{C} 23$ & $-115.1(6)$ \\
\hline $\mathrm{C} 11-\mathrm{C} 12-\mathrm{C} 13-\mathrm{C} 13 \mathrm{~A}$ & $-17.5(7)$ & $\mathrm{CL} 2-\mathrm{PD} 2-\mathrm{C} 22-\mathrm{C} 23$ & $44.5(5)$ \\
\hline $\mathrm{PD} 1-\mathrm{C} 12-\mathrm{C} 13-\mathrm{C} 13 \mathrm{~A}$ & $-83.4(5)$ & $\mathrm{CL} 2^{\mathrm{ii}}-\mathrm{PD} 2-\mathrm{C} 22-\mathrm{C} 23$ & $-165.4(3)$ \\
\hline $\mathrm{C} 11-\mathrm{C} 12-\mathrm{C} 13-\mathrm{PD} 1$ & $66.0(4)$ & $\mathrm{C} 23-\mathrm{PD} 2-\mathrm{C} 22-\mathrm{C} 21$ & $115.1(6)$ \\
\hline $\mathrm{C} 11-\mathrm{PD} 1-\mathrm{C} 13-\mathrm{C} 12$ & $-40.8(4)$ & $\mathrm{CL} 2-\mathrm{PD} 2-\mathrm{C} 22-\mathrm{C} 21$ & $159.6(3)$ \\
\hline $\mathrm{CL} 1-\mathrm{PD} 1-\mathrm{C} 13-\mathrm{C} 12$ & $148.2(4)$ & $\mathrm{CL} 2^{\mathrm{ii}}-\mathrm{PD} 2-\mathrm{C} 22-\mathrm{C} 21$ & $-50.4(5)$ \\
\hline $\mathrm{CL} 1^{\mathrm{i}}-\mathrm{PD} 1-\mathrm{C} 13-\mathrm{C} 12$ & $-54.0(9)$ & $\mathrm{C} 21-\mathrm{C} 22-\mathrm{C} 23-\mathrm{C} 23 \mathrm{~A}$ & $18.3(8)$ \\
\hline $\mathrm{C} 12-\mathrm{PD} 1-\mathrm{C} 13-\mathrm{C} 13 \mathrm{~A}$ & $111.2(6)$ & $\mathrm{PD} 2-\mathrm{C} 22-\mathrm{C} 23-\mathrm{C} 23 \mathrm{~A}$ & $83.4(4)$ \\
\hline $\mathrm{C} 11-\mathrm{PD} 1-\mathrm{C} 13-\mathrm{C} 13 \mathrm{~A}$ & $70.4(4)$ & $\mathrm{C} 21-\mathrm{C} 22-\mathrm{C} 23-\mathrm{PD} 2$ & $-65.1(5)$ \\
\hline $\mathrm{CL} 1-\mathrm{PD} 1-\mathrm{C} 13-\mathrm{C} 13 \mathrm{~A}$ & $-100.6(4)$ & $\mathrm{C} 21-\mathrm{PD} 2-\mathrm{C} 23-\mathrm{C} 22$ & $39.4(4)$ \\
\hline $\mathrm{CL} 11^{\mathrm{i}}-\mathrm{PD} 1-\mathrm{C} 13-\mathrm{C} 13 \mathrm{~A}$ & $57.2(9)$ & $\mathrm{CL} 2-\mathrm{PD} 2-\mathrm{C} 23-\mathrm{C} 22$ & $-150.8(4)$ \\
\hline $\mathrm{C} 12-\mathrm{C} 13-\mathrm{C} 13 \mathrm{~A}-\mathrm{C} 14$ & $-171.2(7)$ & $\mathrm{CL} 2^{\mathrm{ii}}-\mathrm{PD} 2-\mathrm{C} 23-\mathrm{C} 22$ & $46.4(8)$ \\
\hline $\mathrm{PD} 1-\mathrm{C} 13-\mathrm{C} 13 \mathrm{~A}-\mathrm{C} 14$ & $120.3(8)$ & $\mathrm{C} 22-\mathrm{PD} 2-\mathrm{C} 23-\mathrm{C} 23 \mathrm{~A}$ & $-109.2(6)$ \\
\hline $\mathrm{C} 12-\mathrm{C} 13-\mathrm{C} 13 \mathrm{~A}-\mathrm{C} 17 \mathrm{~A}$ & $11.9(7)$ & $\mathrm{C} 21-\mathrm{PD} 2-\mathrm{C} 23-\mathrm{C} 23 \mathrm{~A}$ & $-69.7(4)$ \\
\hline $\mathrm{PD} 1-\mathrm{C} 13-\mathrm{C} 13 \mathrm{~A}-\mathrm{C} 17 \mathrm{~A}$ & $-56.6(5)$ & $\mathrm{CL} 2-\mathrm{PD} 2-\mathrm{C} 23-\mathrm{C} 23 \mathrm{~A}$ & $100.0(4)$ \\
\hline $\mathrm{C} 13-\mathrm{C} 13 \mathrm{~A}-\mathrm{C} 14-\mathrm{C} 15$ & $-177.8(7)$ & $\mathrm{CL} 22^{\mathrm{ii}}-\mathrm{PD} 2-\mathrm{C} 23-\mathrm{C} 23 \mathrm{~A}$ & $-62.8(8)$ \\
\hline $\mathrm{C} 16-\mathrm{C} 17-\mathrm{C} 17 \mathrm{~A}-\mathrm{C} 13 \mathrm{~A}$ & $1.0(9)$ & $\mathrm{C} 22-\mathrm{C} 23-\mathrm{C} 23 \mathrm{~A}-\mathrm{C} 24$ & $170.1(7)$ \\
\hline $\mathrm{C} 16-\mathrm{C} 17-\mathrm{C} 17 \mathrm{~A}-\mathrm{C} 11$ & $-179.3(6)$ & $\mathrm{PD} 2-\mathrm{C} 23-\mathrm{C} 23 \mathrm{~A}-\mathrm{C} 24$ & $-122.9(7)$ \\
\hline $\mathrm{C} 14-\mathrm{C} 13 \mathrm{~A}-\mathrm{C} 17 \mathrm{~A}-\mathrm{C} 17$ & $1.0(9)$ & $\mathrm{C} 22-\mathrm{C} 23-\mathrm{C} 23 \mathrm{~A}-\mathrm{C} 27 \mathrm{~A}$ & $-11.3(7)$ \\
\hline $\mathrm{C} 13-\mathrm{C} 13 \mathrm{~A}-\mathrm{C} 17 \mathrm{~A}-\mathrm{C} 17$ & $178.4(6)$ & $\mathrm{PD} 2-\mathrm{C} 23-\mathrm{C} 23 \mathrm{~A}-\mathrm{C} 27 \mathrm{~A}$ & $55.6(5)$ \\
\hline $\mathrm{C} 14-\mathrm{C} 13 \mathrm{~A}-\mathrm{C} 17 \mathrm{~A}-\mathrm{C} 11$ & $-178.8(6)$ & $\mathrm{C} 23-\mathrm{C} 23 \mathrm{~A}-\mathrm{C} 24-\mathrm{C} 25$ & $178.7(6)$ \\
\hline $\mathrm{C} 13-\mathrm{C} 13 \mathrm{~A}-\mathrm{C} 17 \mathrm{~A}-\mathrm{C} 11$ & $-1.3(7)$ & $\mathrm{C} 26-\mathrm{C} 27-\mathrm{C} 27 \mathrm{~A}-\mathrm{C} 21$ & $-179.3(7)$ \\
\hline $\mathrm{C} 12-\mathrm{C} 11-\mathrm{C} 17 \mathrm{~A}-\mathrm{C} 17$ & $171.2(7)$ & $\mathrm{C} 23-\mathrm{C} 23 \mathrm{~A}-\mathrm{C} 27 \mathrm{~A}-\mathrm{C} 27$ & $-177.8(6)$ \\
\hline $\mathrm{PD} 1-\mathrm{C} 11-\mathrm{C} 17 \mathrm{~A}-\mathrm{C} 17$ & $-122.1(7)$ & $\mathrm{C} 24-\mathrm{C} 23 \mathrm{~A}-\mathrm{C} 27 \mathrm{~A}-\mathrm{C} 21$ & $178.9(6)$ \\
\hline $\mathrm{C} 12-\mathrm{C} 11-\mathrm{C} 17 \mathrm{~A}-\mathrm{C} 13 \mathrm{~A}$ & $-9.1(7)$ & $\mathrm{C} 23-\mathrm{C} 23 \mathrm{~A}-\mathrm{C} 27 \mathrm{~A}-\mathrm{C} 21$ & $0.2(7)$ \\
\hline $\mathrm{PD} 1-\mathrm{C} 11-\mathrm{C} 17 \mathrm{~A}-\mathrm{C} 13 \mathrm{~A}$ & $57.7(5)$ & $\mathrm{C} 22-\mathrm{C} 21-\mathrm{C} 27 \mathrm{~A}-\mathrm{C} 27$ & $-171.5(7)$ \\
\hline $\mathrm{C} 22-\mathrm{PD} 2-\mathrm{CL} 2-\mathrm{PD} 2^{\mathrm{ii}}$ & $157.7(3)$ & $\mathrm{PD} 2-\mathrm{C} 21-\mathrm{C} 27 \mathrm{~A}-\mathrm{C} 27$ & $120.3(7)$ \\
\hline $\mathrm{C} 21-\mathrm{PD} 2-\mathrm{CL} 2-\mathrm{PD} 2^{\mathrm{ii}}$ & $-132.6(8)$ & $\mathrm{C} 22-\mathrm{C} 21-\mathrm{C} 27 \mathrm{~A}-\mathrm{C} 23 \mathrm{~A}$ & $10.8(8)$ \\
\hline $\mathrm{C} 23-\mathrm{PD} 2-\mathrm{CL} 2-\mathrm{PD} 2^{\mathrm{ii}}$ & $-175.51(17)$ & $\mathrm{PD} 2-\mathrm{C} 21-\mathrm{C} 27 \mathrm{~A}-\mathrm{C} 23 \mathrm{~A}$ & $-57.4(5)$ \\
\hline $\mathrm{CL} 2^{\mathrm{ii}}-\mathrm{PD} 2-\mathrm{CL} 2-\mathrm{PD} 2^{\mathrm{ii}}$ & 0 & $\mathrm{C} 32-\mathrm{PD} 3-\mathrm{CL} 3-\mathrm{PD} 3^{\mathrm{iii}}$ & $158.5(3)$ \\
\hline $\mathrm{C} 23-\mathrm{PD} 2-\mathrm{C} 21-\mathrm{C} 22$ & $-38.7(4)$ & C33-PD3-CL3-PD3 ${ }^{\text {iii }}$ & $-138.2(9)$ \\
\hline $\mathrm{CL} 2-\mathrm{PD} 2-\mathrm{C} 21-\mathrm{C} 22$ & $-85.5(9)$ & C31-PD3-CL3-PD3 ${ }^{\mathrm{iii}}$ & $-172.7(2)$ \\
\hline $\mathrm{CL} 22^{\mathrm{ii}}-\mathrm{PD} 2-\mathrm{C} 21-\mathrm{C} 22$ & $143.2(4)$ & CL3 $3^{\mathrm{iii}}-\mathrm{PD} 3-\mathrm{CL} 3-\mathrm{PD} 3^{\mathrm{iii}}$ & 0 \\
\hline $\mathrm{C} 22-\mathrm{PD} 2-\mathrm{C} 21-\mathrm{C} 27 \mathrm{~A}$ & $106.8(6)$ & $\mathrm{C} 33 \mathrm{~A}-\mathrm{PD} 3-\mathrm{CL} 3-\mathrm{PD} 3^{\mathrm{iii}}$ & $-112.5(2)$ \\
\hline $\mathrm{C} 23-\mathrm{PD} 2-\mathrm{C} 21-\mathrm{C} 27 \mathrm{~A}$ & $68.2(4)$ & $\mathrm{C} 33-\mathrm{PD} 3-\mathrm{C} 31-\mathrm{C} 32$ & $39.7(4)$ \\
\hline $\mathrm{CL} 2^{\mathrm{ii}}-\mathrm{PD} 2-\mathrm{C} 21-\mathrm{C} 27 \mathrm{~A}$ & $-110.0(4)$ & $\mathrm{CL} 33^{\mathrm{iii}}-\mathrm{PD} 3-\mathrm{C} 31-\mathrm{C} 32$ & $58.4(9)$ \\
\hline $\mathrm{C} 27 \mathrm{~A}-\mathrm{C} 21-\mathrm{C} 22-\mathrm{C} 23$ & $-17.8(8)$ & CL3-PD3-C31-C32 & $-148.5(4)$ \\
\hline $\mathrm{PD} 2-\mathrm{C} 21-\mathrm{C} 22-\mathrm{C} 23$ & $66.3(5)$ & $\mathrm{C} 33 \mathrm{~A}-\mathrm{PD} 3-\mathrm{C} 31-\mathrm{C} 32$ & $79.2(5)$ \\
\hline $\mathrm{C} 27 \mathrm{~A}-\mathrm{C} 21-\mathrm{C} 22-\mathrm{PD} 2$ & $-84.1(5)$ & $\mathrm{C} 32-\mathrm{PD} 3-\mathrm{C} 31-\mathrm{C} 37 \mathrm{~A}$ & $-110.6(6)$ \\
\hline
\end{tabular}




\begin{tabular}{|c|c|c|c|}
\hline $\mathrm{C} 33-\mathrm{PD} 3-\mathrm{C} 31-\mathrm{C} 37 \mathrm{~A}$ & $-70.9(4)$ & $\mathrm{CL} 3-\mathrm{PD} 3-\mathrm{C} 33 \mathrm{~A}-\mathrm{C} 33$ & $-170.2(4)$ \\
\hline 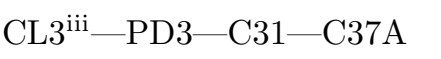 & $-52.3(9)$ & $\mathrm{C} 33-\mathrm{C} 33 \mathrm{~A}-\mathrm{C} 34-\mathrm{C} 35$ & $-178.4(7)$ \\
\hline CL3-PD3-C31-C37A & $100.9(4)$ & $\mathrm{PD} 3-\mathrm{C} 33 \mathrm{~A}-\mathrm{C} 34-\mathrm{C} 35$ & $-99.6(9)$ \\
\hline $\mathrm{C} 33 \mathrm{~A}-\mathrm{PD} 3-\mathrm{C} 31-\mathrm{C} 37 \mathrm{~A}$ & $-31.4(4)$ & $\mathrm{C} 34-\mathrm{C} 35-\mathrm{C} 36-\mathrm{C} 37$ & $0.00(12)$ \\
\hline $\mathrm{C} 37 \mathrm{~A}-\mathrm{C} 31-\mathrm{C} 32-\mathrm{C} 33$ & $17.0(7)$ & $\mathrm{C} 36-\mathrm{C} 37-\mathrm{C} 37 \mathrm{~A}-\mathrm{C} 31$ & $179.0(6)$ \\
\hline $\mathrm{PD} 3-\mathrm{C} 31-\mathrm{C} 32-\mathrm{C} 33$ & $-64.9(4)$ & $\mathrm{C} 33-\mathrm{C} 33 \mathrm{~A}-\mathrm{C} 37 \mathrm{~A}-\mathrm{C} 37$ & $177.8(6)$ \\
\hline $\mathrm{C} 37 \mathrm{~A}-\mathrm{C} 31-\mathrm{C} 32-\mathrm{PD} 3$ & $81.9(4)$ & $\mathrm{PD} 3-\mathrm{C} 33 \mathrm{~A}-\mathrm{C} 37 \mathrm{~A}-\mathrm{C} 37$ & $131.7(6)$ \\
\hline $\mathrm{C} 31-\mathrm{PD} 3-\mathrm{C} 32-\mathrm{C} 33$ & $113.2(6)$ & $\mathrm{C} 34-\mathrm{C} 33 \mathrm{~A}-\mathrm{C} 37 \mathrm{~A}-\mathrm{C} 31$ & $-178.4(6)$ \\
\hline 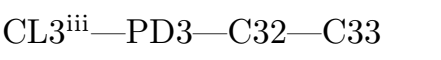 & $-48.1(5)$ & $\mathrm{C} 33-\mathrm{C} 33 \mathrm{~A}-\mathrm{C} 37 \mathrm{~A}-\mathrm{C} 31$ & $-0.5(7)$ \\
\hline $\mathrm{CL} 3-\mathrm{PD} 3-\mathrm{C} 32-\mathrm{C} 33$ & $161.2(3)$ & $\mathrm{PD} 3-\mathrm{C} 33 \mathrm{~A}-\mathrm{C} 37 \mathrm{~A}-\mathrm{C} 31$ & $-46.6(4)$ \\
\hline $\mathrm{C} 33 \mathrm{~A}-\mathrm{PD} 3-\mathrm{C} 32-\mathrm{C} 33$ & $38.1(4)$ & $\mathrm{C} 32-\mathrm{C} 31-\mathrm{C} 37 \mathrm{~A}-\mathrm{C} 37$ & $171.8(7)$ \\
\hline $\mathrm{C} 33-\mathrm{PD} 3-\mathrm{C} 32-\mathrm{C} 31$ & $-113.2(6)$ & $\mathrm{PD} 3-\mathrm{C} 31-\mathrm{C} 37 \mathrm{~A}-\mathrm{C} 37$ & $-121.1(7)$ \\
\hline 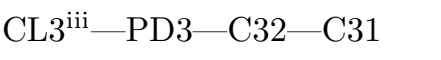 & $-161.3(3)$ & $\mathrm{C} 32-\mathrm{C} 31-\mathrm{C} 37 \mathrm{~A}-\mathrm{C} 33 \mathrm{~A}$ & $-10.2(7)$ \\
\hline CL3-PD3-C32-C31 & $48.0(5)$ & $\mathrm{PD} 3-\mathrm{C} 31-\mathrm{C} 37 \mathrm{~A}-\mathrm{C} 33 \mathrm{~A}$ & $56.9(5)$ \\
\hline $\mathrm{C} 33 \mathrm{~A}-\mathrm{PD} 3-\mathrm{C} 32-\mathrm{C} 31$ & $-75.0(4)$ & $\mathrm{C} 2-\mathrm{PD}-\mathrm{CL}-\mathrm{PD}^{\mathrm{iv}}$ & $159.2(3)$ \\
\hline $\mathrm{C} 31-\mathrm{C} 32-\mathrm{C} 33-\mathrm{C} 33 \mathrm{~A}$ & $-17.5(7)$ & $\mathrm{C} 3-\mathrm{PD}-\mathrm{CL}-\mathrm{PD}^{\mathrm{iv}}$ & $-172.18(18)$ \\
\hline PD3-C32-C33-C33A & $-83.6(5)$ & $\mathrm{CL}^{\mathrm{iv}}-\mathrm{PD}-\mathrm{CL}-\mathrm{PD}^{\mathrm{iv}}$ & 0 \\
\hline $\mathrm{C} 31-\mathrm{C} 32-\mathrm{C} 33-\mathrm{PD} 3$ & $66.2(4)$ & $\mathrm{C} 7 \mathrm{~A}-\mathrm{PD}-\mathrm{CL}-\mathrm{PD}^{\mathrm{iv}}$ & $-111.2(2)$ \\
\hline $\mathrm{C} 31-\mathrm{PD} 3-\mathrm{C} 33-\mathrm{C} 32$ & $-40.1(4)$ & $\mathrm{C} 3 \mathrm{~A}-\mathrm{PD}-\mathrm{CL}-\mathrm{PD}^{\mathrm{iv}}$ & $-136.48(15)$ \\
\hline $\mathrm{C} 33 \mathrm{~A}-\mathrm{PD} 3-\mathrm{C} 33-\mathrm{C} 32$ & $-111.1(6)$ & $\mathrm{C} 3-\mathrm{PD}-\mathrm{C} 1-\mathrm{C} 2$ & $-39.1(4)$ \\
\hline $\mathrm{C} 32-\mathrm{PD} 3-\mathrm{C} 33-\mathrm{C} 33 \mathrm{~A}$ & $111.1(6)$ & $\mathrm{CL}^{\mathrm{iv}}-\mathrm{PD}-\mathrm{C} 1-\mathrm{C} 2$ & $146.8(3)$ \\
\hline $\mathrm{C} 31-\mathrm{PD} 3-\mathrm{C} 33-\mathrm{C} 33 \mathrm{~A}$ & $71.0(4)$ & $\mathrm{C} 7 \mathrm{~A}-\mathrm{PD}-\mathrm{C} 1-\mathrm{C} 2$ & $-110.1(6)$ \\
\hline $\mathrm{C} 32-\mathrm{C} 33-\mathrm{C} 33 \mathrm{~A}-\mathrm{C} 34$ & $-171.1(7)$ & $\mathrm{C} 3 \mathrm{~A}-\mathrm{PD}-\mathrm{C} 1-\mathrm{C} 2$ & $-77.9(4)$ \\
\hline $\mathrm{PD} 3-\mathrm{C} 33-\mathrm{C} 33 \mathrm{~A}-\mathrm{C} 34$ & $119.1(8)$ & $\mathrm{C} 2-\mathrm{PD}-\mathrm{C} 1-\mathrm{C} 7 \mathrm{~A}$ & $110.1(6)$ \\
\hline $\mathrm{C} 32-\mathrm{C} 33-\mathrm{C} 33 \mathrm{~A}-\mathrm{C} 37 \mathrm{~A}$ & $11.3(7)$ & $\mathrm{C} 3-\mathrm{PD}-\mathrm{C} 1-\mathrm{C} 7 \mathrm{~A}$ & $71.0(4)$ \\
\hline PD3-C33-C33A-C37A & $-58.4(5)$ & $\mathrm{C} 3 \mathrm{~A}-\mathrm{PD}-\mathrm{C} 1-\mathrm{C} 7 \mathrm{~A}$ & $32.2(4)$ \\
\hline $\mathrm{C} 32-\mathrm{C} 33-\mathrm{C} 33 \mathrm{~A}-\mathrm{PD} 3$ & $69.7(5)$ & $\mathrm{C} 7 \mathrm{~A}-\mathrm{C} 1-\mathrm{C} 2-\mathrm{C} 3$ & $-17.6(7)$ \\
\hline $\mathrm{C} 32-\mathrm{PD} 3-\mathrm{C} 33 \mathrm{~A}-\mathrm{C} 34$ & $-162.1(9)$ & $\mathrm{PD}-\mathrm{C} 1-\mathrm{C} 2-\mathrm{C} 3$ & $65.2(4)$ \\
\hline $\mathrm{C} 31-\mathrm{PD} 3-\mathrm{C} 33 \mathrm{~A}-\mathrm{C} 34$ & $152.2(9)$ & $\mathrm{C} 7 \mathrm{~A}-\mathrm{C} 1-\mathrm{C} 2-\mathrm{PD}$ & $-82.8(5)$ \\
\hline $\mathrm{CL} 3^{\mathrm{iii}}-\mathrm{PD} 3-\mathrm{C} 33 \mathrm{~A}-\mathrm{C} 34$ & $-33.8(9)$ & $\mathrm{C} 1-\mathrm{PD}-\mathrm{C} 2-\mathrm{C} 3$ & $-114.3(6)$ \\
\hline CL3-PD3-C33A-C34 & $70.1(9)$ & $\mathrm{CL}^{\mathrm{iv}}-\mathrm{PD}-\mathrm{C} 2-\mathrm{C} 3$ & $-160.7(3)$ \\
\hline $\mathrm{C} 32-\mathrm{PD} 3-\mathrm{C} 33 \mathrm{~A}-\mathrm{C} 37 \mathrm{~A}$ & $80.0(4)$ & $\mathrm{CL}-\mathrm{PD}-\mathrm{C} 2-\mathrm{C} 3$ & $47.9(5)$ \\
\hline $\mathrm{C} 33-\mathrm{PD} 3-\mathrm{C} 33 \mathrm{~A}-\mathrm{C} 37 \mathrm{~A}$ & $122.4(6)$ & $\mathrm{C} 7 \mathrm{~A}-\mathrm{PD}-\mathrm{C} 2-\mathrm{C} 3$ & $-75.4(4)$ \\
\hline $\mathrm{C} 31-\mathrm{PD} 3-\mathrm{C} 33 \mathrm{~A}-\mathrm{C} 37 \mathrm{~A}$ & $34.3(4)$ & $\mathrm{C} 3 \mathrm{~A}-\mathrm{PD}-\mathrm{C} 2-\mathrm{C} 3$ & $-38.0(4)$ \\
\hline $\mathrm{CL} 33^{\mathrm{iii}}-\mathrm{PD} 3-\mathrm{C} 33 \mathrm{~A}-\mathrm{C} 37 \mathrm{~A}$ & $-151.7(4)$ & $\mathrm{C} 3-\mathrm{PD}-\mathrm{C} 2-\mathrm{C} 1$ & $114.3(6)$ \\
\hline $\mathrm{CL} 3-\mathrm{PD} 3-\mathrm{C} 33 \mathrm{~A}-\mathrm{C} 37 \mathrm{~A}$ & $-47.8(5)$ & $\mathrm{CL}^{\mathrm{iv}}-\mathrm{PD}-\mathrm{C} 2-\mathrm{C} 1$ & $-46.3(4)$ \\
\hline $\mathrm{C} 32-\mathrm{PD} 3-\mathrm{C} 33 \mathrm{~A}-\mathrm{C} 33$ & $-42.4(4)$ & $\mathrm{CL}-\mathrm{PD}-\mathrm{C} 2-\mathrm{C} 1$ & $162.2(3)$ \\
\hline $\mathrm{C} 31-\mathrm{PD} 3-\mathrm{C} 33 \mathrm{~A}-\mathrm{C} 33$ & $-88.1(5)$ & $\mathrm{C} 7 \mathrm{~A}-\mathrm{PD}-\mathrm{C} 2-\mathrm{C} 1$ & $38.9(4)$ \\
\hline 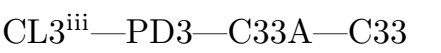 & $85.9(4)$ & $\mathrm{C} 3 \mathrm{~A}-\mathrm{PD}-\mathrm{C} 2-\mathrm{C} 1$ & $76.3(4)$ \\
\hline
\end{tabular}




\begin{tabular}{|c|c|c|c|}
\hline $\mathrm{C} 1-\mathrm{C} 2-\mathrm{C} 3-\mathrm{C} 3 \mathrm{~A}$ & $18.2(7)$ & $\mathrm{C} 7 \mathrm{~A}-\mathrm{PD}-\mathrm{C} 3 \mathrm{~A}-\mathrm{C} 3$ & $124.4(6)$ \\
\hline $\mathrm{PD}-\mathrm{C} 2-\mathrm{C} 3-\mathrm{C} 3 \mathrm{~A}$ & $82.1(5)$ & $\mathrm{C} 3-\mathrm{C} 3 \mathrm{~A}-\mathrm{C} 4-\mathrm{C} 5$ & $178.4(7)$ \\
\hline $\mathrm{C} 1-\mathrm{C} 2-\mathrm{C} 3-\mathrm{PD}$ & $-63.9(4)$ & $\mathrm{PD}-\mathrm{C} 3 \mathrm{~A}-\mathrm{C} 4-\mathrm{C} 5$ & $96.7(8)$ \\
\hline $\mathrm{C} 1-\mathrm{PD}-\mathrm{C} 3-\mathrm{C} 2$ & $39.6(4)$ & $\mathrm{C} 6-\mathrm{C} 7-\mathrm{C} 7 \mathrm{~A}-\mathrm{C} 1$ & $-179.0(7)$ \\
\hline $\mathrm{CL}^{\mathrm{iv}}-\mathrm{PD}-\mathrm{C} 3-\mathrm{C} 2$ & $60.5(8)$ & $\mathrm{C} 6-\mathrm{C} 7-\mathrm{C} 7 \mathrm{~A}-\mathrm{PD}$ & $-100.4(8)$ \\
\hline $\mathrm{CL}-\mathrm{PD}-\mathrm{C} 3-\mathrm{C} 2$ & $-148.3(4)$ & $\mathrm{C} 3-\mathrm{C} 3 \mathrm{~A}-\mathrm{C} 7 \mathrm{~A}-\mathrm{C} 7$ & $-177.2(6)$ \\
\hline $\mathrm{C} 7 \mathrm{~A}-\mathrm{PD}-\mathrm{C} 3-\mathrm{C} 2$ & $79.7(4)$ & $\mathrm{PD}-\mathrm{C} 3 \mathrm{~A}-\mathrm{C} 7 \mathrm{~A}-\mathrm{C} 7$ & $-131.3(7)$ \\
\hline $\mathrm{C} 3 \mathrm{~A}-\mathrm{PD}-\mathrm{C} 3-\mathrm{C} 2$ & $110.7(6)$ & $\mathrm{C} 4-\mathrm{C} 3 \mathrm{~A}-\mathrm{C} 7 \mathrm{~A}-\mathrm{C} 1$ & $179.0(6)$ \\
\hline $\mathrm{C} 2-\mathrm{PD}-\mathrm{C} 3-\mathrm{C} 3 \mathrm{~A}$ & $-110.7(6)$ & $\mathrm{C} 3-\mathrm{C} 3 \mathrm{~A}-\mathrm{C} 7 \mathrm{~A}-\mathrm{C} 1$ & $0.8(7)$ \\
\hline $\mathrm{C} 1-\mathrm{PD}-\mathrm{C} 3-\mathrm{C} 3 \mathrm{~A}$ & $-71.1(4)$ & $\mathrm{PD}-\mathrm{C} 3 \mathrm{~A}-\mathrm{C} 7 \mathrm{~A}-\mathrm{C} 1$ & $46.7(4)$ \\
\hline $\mathrm{CL}^{\mathrm{iv}}-\mathrm{PD}-\mathrm{C} 3-\mathrm{C} 3 \mathrm{~A}$ & $-50.2(8)$ & $\mathrm{C} 4-\mathrm{C} 3 \mathrm{~A}-\mathrm{C} 7 \mathrm{~A}-\mathrm{PD}$ & $132.3(6)$ \\
\hline $\mathrm{CL}-\mathrm{PD}-\mathrm{C} 3-\mathrm{C} 3 \mathrm{~A}$ & $101.0(4)$ & $\mathrm{C} 3-\mathrm{C} 3 \mathrm{~A}-\mathrm{C} 7 \mathrm{~A}-\mathrm{PD}$ & $-45.9(4)$ \\
\hline $\mathrm{C} 7 \mathrm{~A}-\mathrm{PD}-\mathrm{C} 3-\mathrm{C} 3 \mathrm{~A}$ & $-31.0(3)$ & $\mathrm{C} 2-\mathrm{C} 1-\mathrm{C} 7 \mathrm{~A}-\mathrm{C} 7$ & $-171.9(8)$ \\
\hline $\mathrm{C} 2-\mathrm{C} 3-\mathrm{C} 3 \mathrm{~A}-\mathrm{C} 4$ & $170.3(7)$ & $\mathrm{PD}-\mathrm{C} 1-\mathrm{C} 7 \mathrm{~A}-\mathrm{C} 7$ & $118.9(8)$ \\
\hline $\mathrm{PD}-\mathrm{C} 3-\mathrm{C} 3 \mathrm{~A}-\mathrm{C} 4$ & $-122.1(7)$ & $\mathrm{C} 2-\mathrm{C} 1-\mathrm{C} 7 \mathrm{~A}-\mathrm{C} 3 \mathrm{~A}$ & $10.4(7)$ \\
\hline $\mathrm{C} 2-\mathrm{C} 3-\mathrm{C} 3 \mathrm{~A}-\mathrm{C} 7 \mathrm{~A}$ & $-11.8(7)$ & $\mathrm{PD}-\mathrm{C} 1-\mathrm{C} 7 \mathrm{~A}-\mathrm{C} 3 \mathrm{~A}$ & $-58.8(5)$ \\
\hline $\mathrm{PD}-\mathrm{C} 3-\mathrm{C} 3 \mathrm{~A}-\mathrm{C} 7 \mathrm{~A}$ & $55.8(5)$ & $\mathrm{C} 2-\mathrm{C} 1-\mathrm{C} 7 \mathrm{~A}-\mathrm{PD}$ & $69.2(5)$ \\
\hline $\mathrm{C} 2-\mathrm{C} 3-\mathrm{C} 3 \mathrm{~A}-\mathrm{PD}$ & $-67.6(4)$ & $\mathrm{C} 2-\mathrm{PD}-\mathrm{C} 7 \mathrm{~A}-\mathrm{C} 7$ & $-162.4(8)$ \\
\hline $\mathrm{C} 2-\mathrm{PD}-\mathrm{C} 3 \mathrm{~A}-\mathrm{C} 4$ & $162.5(8)$ & $\mathrm{C} 1-\mathrm{PD}-\mathrm{C} 7 \mathrm{~A}-\mathrm{C} 7$ & $-119.3(9)$ \\
\hline $\mathrm{C} 1-\mathrm{PD}-\mathrm{C} 3 \mathrm{~A}-\mathrm{C} 4$ & $-151.5(8)$ & $\mathrm{C} 3-\mathrm{PD}-\mathrm{C} 7 \mathrm{~A}-\mathrm{C} 7$ & $152.5(8)$ \\
\hline $\mathrm{C} 3-\mathrm{PD}-\mathrm{C} 3 \mathrm{~A}-\mathrm{C} 4$ & $119.7(8)$ & $\mathrm{CL}^{\mathrm{iv}}-\mathrm{PD}-\mathrm{C} 7 \mathrm{~A}-\mathrm{C} 7$ & $-33.1(7)$ \\
\hline $\mathrm{CL}^{\mathrm{iv}}-\mathrm{PD}-\mathrm{C} 3 \mathrm{~A}-\mathrm{C} 4$ & $-77.9(7)$ & $\mathrm{CL}-\mathrm{PD}-\mathrm{C} 7 \mathrm{~A}-\mathrm{C} 7$ & $69.8(8)$ \\
\hline $\mathrm{CL}-\mathrm{PD}-\mathrm{C} 3 \mathrm{~A}-\mathrm{C} 4$ & $28.5(7)$ & $\mathrm{C} 3 \mathrm{~A}-\mathrm{PD}-\mathrm{C} 7 \mathrm{~A}-\mathrm{C} 7$ & $119.4(9)$ \\
\hline $\mathrm{C} 7 \mathrm{~A}-\mathrm{PD}-\mathrm{C} 3 \mathrm{~A}-\mathrm{C} 4$ & $-115.9(8)$ & $\mathrm{C} 2-\mathrm{PD}-\mathrm{C} 7 \mathrm{~A}-\mathrm{C} 3 \mathrm{~A}$ & $78.2(4)$ \\
\hline $\mathrm{C} 2-\mathrm{PD}-\mathrm{C} 3 \mathrm{~A}-\mathrm{C} 7 \mathrm{~A}$ & $-81.5(4)$ & $\mathrm{C} 1-\mathrm{PD}-\mathrm{C} 7 \mathrm{~A}-\mathrm{C} 3 \mathrm{~A}$ & $121.3(6)$ \\
\hline $\mathrm{C} 1-\mathrm{PD}-\mathrm{C} 3 \mathrm{~A}-\mathrm{C} 7 \mathrm{~A}$ & $-35.6(4)$ & $\mathrm{C} 3-\mathrm{PD}-\mathrm{C} 7 \mathrm{~A}-\mathrm{C} 3 \mathrm{~A}$ & $33.1(4)$ \\
\hline $\mathrm{C} 3-\mathrm{PD}-\mathrm{C} 3 \mathrm{~A}-\mathrm{C} 7 \mathrm{~A}$ & $-124.4(6)$ & $\mathrm{CL}^{\mathrm{iv}}-\mathrm{PD}-\mathrm{C} 7 \mathrm{~A}-\mathrm{C} 3 \mathrm{~A}$ & $-152.5(3)$ \\
\hline $\mathrm{CL}^{\mathrm{iv}}-\mathrm{PD}-\mathrm{C} 3 \mathrm{~A}-\mathrm{C} 7 \mathrm{~A}$ & $38.0(4)$ & $\mathrm{CL}-\mathrm{PD}-\mathrm{C} 7 \mathrm{~A}-\mathrm{C} 3 \mathrm{~A}$ & $-49.6(5)$ \\
\hline $\mathrm{CL}-\mathrm{PD}-\mathrm{C} 3 \mathrm{~A}-\mathrm{C} 7 \mathrm{~A}$ & $144.5(3)$ & $\mathrm{C} 2-\mathrm{PD}-\mathrm{C} 7 \mathrm{~A}-\mathrm{C} 1$ & $-43.1(4)$ \\
\hline $\mathrm{C} 2-\mathrm{PD}-\mathrm{C} 3 \mathrm{~A}-\mathrm{C} 3$ & $42.9(4)$ & $\mathrm{C} 3-\mathrm{PD}-\mathrm{C} 7 \mathrm{~A}-\mathrm{C} 1$ & $-88.2(5)$ \\
\hline $\mathrm{C} 1-\mathrm{PD}-\mathrm{C} 3 \mathrm{~A}-\mathrm{C} 3$ & $88.8(4)$ & $\mathrm{CL}^{\mathrm{iv}}-\mathrm{PD}-\mathrm{C} 7 \mathrm{~A}-\mathrm{C} 1$ & $86.3(4)$ \\
\hline $\mathrm{CL}^{\mathrm{iv}}-\mathrm{PD}-\mathrm{C} 3 \mathrm{~A}-\mathrm{C} 3$ & $162.4(3)$ & $\mathrm{CL}-\mathrm{PD}-\mathrm{C} 7 \mathrm{~A}-\mathrm{C} 1$ & $-170.9(4)$ \\
\hline $\mathrm{CL}-\mathrm{PD}-\mathrm{C} 3 \mathrm{~A}-\mathrm{C} 3$ & $-91.1(4)$ & $\mathrm{C} 3 \mathrm{~A}-\mathrm{PD}-\mathrm{C} 7 \mathrm{~A}-\mathrm{C} 1$ & $-121.3(6)$ \\
\hline
\end{tabular}

Symmetry codes: (i) $1-x, 2-y,-z$; (ii) $1-x, 1-y,-z$; (iii) $2-x, 2-y,-z$; (iv) $2-x, 1-y, 2-z$. 
All non-H atoms were refined by full-matrix least-squares with anisotropic displacement parameters while $\mathrm{H}$ atoms were placed in idealized position.

Data collection: SAINT (Bruker, 1999). Cell refinement: SMART (Bruker, 1999). Data reduction: SAINT (Bruker, 1999). Program(s) used to solve structure: SHELXS97 (Sheldrick, 1997). Program(s) used to refine structure: SHELXL97 (Sheldrick, 1997). Molecular graphics: XP (Bruker, 1999). Software used to prepare material for publication: UdMX (local program).

The financial supports of the Natural Sciences and Engineering Research Council of Canada and the Fonds FCAR du Ministère de l'Éducation du Québec are gratefully acknowledged. We are also indebted to Johnson Matthey for generous gift of $\mathrm{PdCl}_{2}$.

Supplementary data for this paper are available from the IUCr electronic archives (Reference: ). Services for accessing these data are described at the back of the journal.

\section{References}

Bruker (1997). SHELXTL Release 5.10; The Complete Software Package for Single Crystal Structure Determination, Bruker AXS Inc., Madison,USA.

Bruker (1999). SAINT Release 6.06; Integration Software for Single Crystal Data, Bruker AXS Inc., Madison,USA.

Bruker (1999). SMART Release 5.059; Bruker Molecular Analysis Research Tool, Bruker AXS Inc., Madison, USA.

Sheldrick, G. M. (1996). SADABS, Bruker Area Detector Absorption Corrections, Bruker AXS Inc., Madison, USA.

Sheldrick, G. M. (1997). SHELXS97. Program for the Solution of Crystal Structures. University of Gottingen, Germany.

Sheldrick, G. M. (1997). SHELXL97. Program for the Refinement of Crystal Structures. University of Gottingen, Germany.

Spek, A. L. (1995). PLATON, July 1995 version; Molecular Geometry Program, University of Utrecht, The Netherlands.;

Fig 1 ORTEP view of the title compound. Thermal ellipsoids are shown at $30 \%$ probability levels.

Table 1. Selected geometric parameters $\left(\stackrel{\circ}{\circ}{ }^{\circ}\right)$ for the title compound. 


\section{Supplementary data}

The tables of data shown below are not normally printed in Acta Cryst. Section $C$ but the data will be available electronically via the online contents pages at

\section{http://journals.iucr.org/c/journalhomepage.html}

Table S1. Fractional atomic coordinates and equivalent isotropic displacement parameters $\left(\AA^{2}\right)$

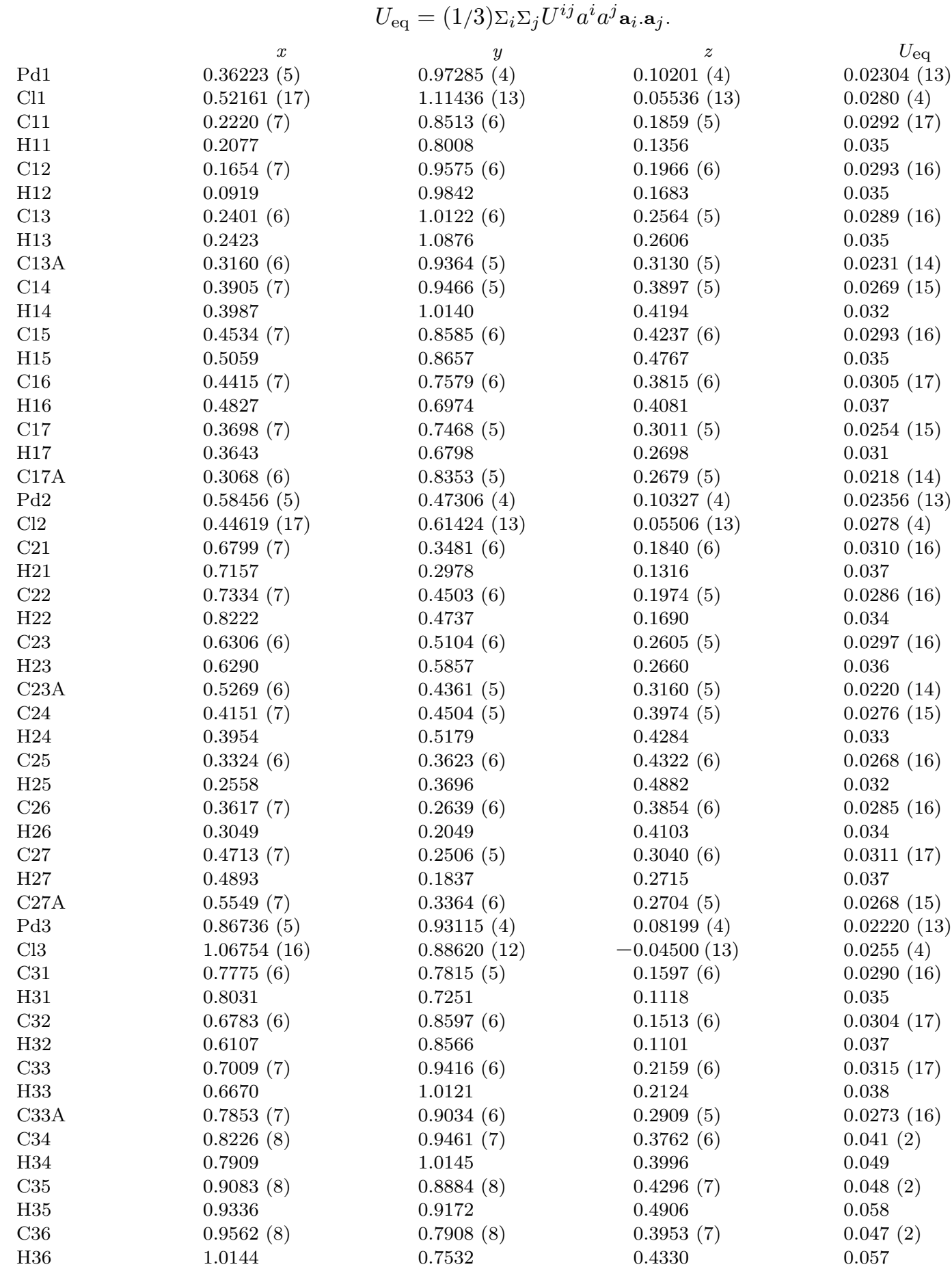




$\begin{array}{ll}\text { C37 } & 0.9218(7) \\ \mathrm{H} 37 & 0.9566 \\ \mathrm{C} 37 \mathrm{~A} & 0.8348(7) \\ \mathrm{Pd} & 0.90660(5) \\ \mathrm{Cl} & 1.04292(16) \\ \mathrm{C} 1 & 0.8066(7) \\ \mathrm{H} 1 & 0.7707 \\ \mathrm{C} 2 & 0.7513(6) \\ \mathrm{H} 2 & 0.6626 \\ \mathrm{C} 3 & 0.8545(6) \\ \mathrm{H} 3 & 0.8561 \\ \mathrm{C} 3 \mathrm{~A} & 0.9589(6) \\ \mathrm{C} 4 & 1.0707(7) \\ \mathrm{H} 4 & 1.0912 \\ \mathrm{C} 5 & 1.1518(7) \\ \mathrm{H} 5 & 1.2274 \\ \mathrm{C} 6 & 1.1249(7) \\ \mathrm{H} 6 & 1.1833(8) \\ \mathrm{C} 7 & 1.0136(8) \\ \mathrm{H} 7 & 0.9955 \\ \mathrm{C} 7 \mathrm{~A} & 0.9314(7)\end{array}$

$0.7450(6)$
0.6778
$0.8021(6)$
$0.57178(4)$
$0.61170(12)$
$0.5643(6)$
0.4946
$0.6481(6)$
0.6524
$0.7239(5)$
0.7783
$0.7053(5)$
$0.7630(6)$
0.8299
$0.7192(7)$
0.7582
$0.6196(7)$
0.5915
$0.5611(6)$
0.4931
$0.6038(6)$

$0.3062(6)$
0.2817
$0.2554(5)$
$0.92087(4)$
$1.04848(13)$
$0.7874(6)$
0.7894
$0.8543(5)$
0.8953
$0.8480(5)$
0.8977
$0.7521(5)$
$0.7009(6)$
0.7262
$0.6110(6)$
0.5734
$0.5748(6)$
0.5142
$0.6265(6)$
0.6022
$0.7127(5)$

$0.0350(18)$

0.042

0.0271 (16)

$0.02124(12)$

0.0255 (3)

0.0298 (17)

0.036

0.0274 (15)

0.033

0.0253 (14)

0.030

0.0207 (14)

0.0301 (17)

0.036

0.0367 (19)

0.044

0.0377 (19)

0.045

0.0353 (18)

0.042

0.0267 (15)

Table S2. Anisotropic displacement parameters $\left(\AA^{2}\right)$

$\operatorname{Pd} 1$

$U_{11}$

$0.0266(3)$

$U_{22}$

$0.0196(3)$

0.0379 (9)

0.0187 (8)

0.037 (4)

0.029 (4)

C12

C13

$\mathrm{C} 13 \mathrm{~A}$

C14

0.027 (4)

$0.020(3)$

0.033 (4)

C15

C16

C17

C17A

0.033 (4)

0.036 (4)

0.036 (4)

0.024 (3)

$\mathrm{Pd} 2$

$\mathrm{Cl} 2$

C21

$\mathrm{C} 22$

C23

$\mathrm{C} 23 \mathrm{~A}$

C24

C25

C26

C27

C27A

Pd3

$\mathrm{Cl} 3$

C31

C32

C33

C33A

C34

C35

C36

C37

C37A

$\mathrm{Pd}$

$0.0273(3)$

$0.0362(9)$

0.039 (4)

0.024 (4)

0.034 (4)

$0.023(3)$

0.038 (4)

$0.023(3)$

$0.038(4)$

$0.045(4)$

$0.025(4)$

$0.0241(3)$

0.032 (4)

0.028 (4)

0.026 (4)

0.021 (3)

0.020 (3)

0.029 (4)

0.023 (4)

0.015 (3)

$0.021(3)$

0.0196 (3)

$0.0190(8)$

$0.024(4)$

0.036 (4)

0.026 (4)

0.024 (4)

0.018 (3)

$0.030(4)$

0.019 (3)

$0.015(3)$

0.034 (4)

$0.0174(3)$

0.0315 (9)

0.029 (4)

$0.022(3)$

$0.0153(7)$

0.023 (4)

0.034 (4)

0.025 (4)

0.028 (4)

0.027 (4)

0.053 (5)

0.054 (5)

0.038 (5)

0.033 (4)

0.039 (5)

$0.065(7)$

$0.070(7)$

0.031 (4)

0.029 (4)

$0.0165(3)$

0.0247 (3)

0.0328 (9)

$0.0167(7)$

0.021 (4)

$\begin{array}{ll}\text { C2 } & 0.019(3) \\ \text { C3 } & 0.036(4) \\ \text { C3A } & 0.021(3)\end{array}$

$\begin{array}{ll}\text { C2 } & 0.019(3) \\ \text { C3 } & 0.036(4) \\ \text { C3A } & 0.021(3)\end{array}$

C4 $0.035(4)$

$0.032(4)$

$0.017(3)$

$0.017(3)$

$0.025(4)$

\begin{tabular}{l}
$U_{33}$ \\
$0.0225(3)$ \\
$0.0267(9)$ \\
$0.019(3)$ \\
$0.032(4)$ \\
$0.031(4)$ \\
$0.027(4)$ \\
$0.028(4)$ \\
$0.026(4)$ \\
$0.032(4)$ \\
$0.021(4)$ \\
$0.019(3)$ \\
$0.0236(3)$ \\
$0.0280(9)$ \\
$0.031(4)$ \\
$0.024(4)$ \\
$0.032(4)$ \\
$0.020(3)$ \\
$0.027(4)$ \\
$0.024(4)$ \\
$0.028(4)$ \\
$0.035(4)$ \\
$0.022(4)$ \\
$0.0243(3)$ \\
$0.0282(9)$ \\
$0.032(4)$ \\
$0.035(4)$ \\
$0.035(4)$ \\
$0.021(4)$ \\
$0.026(4)$ \\
$0.027(4)$ \\
$0.035(5)$ \\
$0.037(5)$ \\
$0.021(4)$ \\
$0.0226(3)$ \\
$0.0291(9)$ \\
$0.033(4)$ \\
$0.030(4)$ \\
$0.026(4)$ \\
$0.024(3)$ \\
$0.031(4)$ \\
\hline
\end{tabular}

$U_{12} \quad U_{13}$

$-0.0011(2) \quad-0.0046(2)$

$U_{23}$

$0.0022(2)$

$-0.0008(6)$

$0.005(3)$

$-0.016(3) \quad-0.005(3)$

$\begin{array}{lll}0.002(3) & -0.009(3) & 0.002(3) \\ 0.006(3) & -0.002(3) & 0.008(3)\end{array}$

$0.002(3) \quad-0.002(3) \quad-0.001(3)$

$-0.005(3) \quad-0.007(3)$

$-0.005(3) \quad-0.008(3)$

$0.005(3) \quad-0.008(3)$

$-0.005(3) \quad 0.002(3)$

$0.002(3) \quad-0.001(3)$

$0.0015(2) \quad-0.0055(2)$

$0.0065(7) \quad-0.0063(7)$

$0.016(3) \quad-0.010(3)$

$-0.003(3) \quad-0.001(3)$

$-0.005(3) \quad-0.014(3)$

$-0.003(3) \quad-0.009(3)$

$0.005(3) \quad-0.008(3)$

$0.003(3) \quad 0.002(3)$

$0.002(3)$

$0.005(3)$

0.009 (3)

$0.002(3)$

$0.001(3)$

$0.0033(2)$

$0.0021(7)$

$0.000(3)$

$0.005(3)$

$0.000(3)$

$0.005(3)$

$-0.003(3)$

$\begin{array}{lll}-0.005(3) & -0.008(3) & 0.009(3)\end{array}$

$0.004(3) \quad-0.014(3) \quad 0.007(3)$

$0.002(3) \quad-0.007(3) \quad 0.005(3)$

$-0.0001(2) \quad-0.0036(2)$

$0.0008(7) \quad-0.0033(7)$

$-0.002(3) \quad 0.001(3)$

$-0.002(3) \quad-0.005(3)$

$0.010(3) \quad-0.002(3)$

$-0.003(3) \quad 0.009(3)$

$-0.013(4) \quad 0.005(4)$

$-0.023(5) \quad-0.010(4)$

$-0.009(4) \quad-0.016(4)$

$0.009(3) \quad 0.000(3)$

$0.004(3)$

$-0.003(3)$

$-0.0055(2)$

$0.0012(7) \quad-0.0112(7)$

$-0.009(3) \quad-0.017(3)$

$0.003(3) \quad-0.005(3)$

$0.007(3) \quad-0.013(3)$

$0.001(3) \quad-0.005(3)$

$0.0039(2)$

0.0008 (6)

0.002 (3)

0.015 (3)

0.006 (3)

$0.002(3)$

0.002 (4)

0.006 (4)

0.021 (5)

0.011 (3)

0.010 (3)

$0.0030(2)$

0.0000 (6)

0.014 (3)

0.007 (3)

$0.005(3)$

$0.003(3)$

0.008 (3) 


$\begin{array}{lllllll}\text { C5 } & 0.024(4) & 0.047(5) & 0.037(5) & -0.002(3) & -0.005(3) & 0.015(4) \\ \text { C6 } & 0.039(4) & 0.044(5) & 0.029(4) & 0.013(4) & -0.006(3) & 0.004(4) \\ \text { C7 } & 0.054(5) & 0.027(4) & 0.030(4) & 0.006(4) & -0.019(4) & 0.002(3) \\ \text { C7A } & 0.035(4) & 0.027(4) & 0.020(3) & 0.004(3) & -0.010(3) & 0.001(3)\end{array}$

Pd1-C12

$\mathrm{Pd} 1-\mathrm{C} 13$

$\mathrm{Pd} 1-\mathrm{C} 11$

$\mathrm{Pd} 1-\mathrm{Cl1}$

$\mathrm{Pd} 1-\mathrm{Cl}^{\mathrm{i}}$

$\mathrm{Cl} 1-\mathrm{Pd} 1^{\mathrm{i}}$

C11-C12

C11-C17a

C11-H11

C12-C13

C12-H12

C13-C13a

C13-H13

$\mathrm{C} 13 \mathrm{a}-\mathrm{C} 14$

$\mathrm{C} 13 \mathrm{a}-\mathrm{C} 17 \mathrm{a}$

C14-C15

C14-H14

C15-C16

C15-H15

C16-C17

C16-H16

$\mathrm{C} 17-\mathrm{C} 17 \mathrm{a}$

C17-H17

$\mathrm{Pd} 2-\mathrm{C} 22$

$\mathrm{Pd} 2-\mathrm{C} 21$

$\mathrm{Pd} 2-\mathrm{C} 23$

$\mathrm{Pd} 2-\mathrm{Cl} 2$

$\mathrm{Pd} 2-\mathrm{Cl} 2{ }^{\mathrm{ii}}$

$\mathrm{Cl} 2-\mathrm{Pd} 2{ }^{\mathrm{ii}}$

C21-C22

$\mathrm{C} 21-\mathrm{C} 27 \mathrm{a}$

C21-H21

C22-C23

$\mathrm{C} 22-\mathrm{H} 22$

C23-C23a

$\mathrm{C} 23-\mathrm{H} 23$

$\mathrm{C} 23 \mathrm{a}-\mathrm{C} 24$

$\mathrm{C} 23 \mathrm{a}-\mathrm{C} 27 \mathrm{a}$

C24-C25

$\mathrm{C} 24-\mathrm{H} 24$

$\mathrm{C} 25-\mathrm{C} 26$

$\mathrm{C} 25-\mathrm{H} 25$

$\mathrm{C} 26-\mathrm{C} 27$

$\mathrm{C} 26-\mathrm{H} 26$

$\mathrm{C} 27-\mathrm{C} 27 \mathrm{a}$

$\mathrm{C} 27-\mathrm{H} 27$

Pd3-C32

Pd3-C33

C12-PD1-C13

C12-PD1-C11

C13-PD1-C11

C12-PD1-CL1

C13-PD1-CL1

C11-PD1-CL1

C12-PD1-CL1 ${ }^{\mathrm{i}}$

Table S3. Geometric parameters $\left(\stackrel{\circ}{\circ}{ }^{\circ}\right)$

\begin{tabular}{|c|c|c|}
\hline $2.127(7)$ & $\mathrm{Pd} 3-\mathrm{C} 31$ & $2.208(6)$ \\
\hline $2.172(7)$ & $\mathrm{Pd} 3-\mathrm{Cl} 3^{\mathrm{iii}}$ & $2.3962(16)$ \\
\hline $2.194(6)$ & $\mathrm{Pd} 3-\mathrm{Cl} 3$ & $2.4086(17)$ \\
\hline $2.3950(16)$ & $\mathrm{Pd} 3-\mathrm{C} 33 \mathrm{a}$ & $2.631(6)$ \\
\hline $2.4035(17)$ & $\mathrm{Cl} 3-\mathrm{Pd} 3^{\mathrm{iii}}$ & $2.3962(16)$ \\
\hline $2.4035(17)$ & C31-C32 & $1.421(9)$ \\
\hline $1.447(10)$ & $\mathrm{C} 31-\mathrm{C} 37 \mathrm{a}$ & $1.485(10)$ \\
\hline $1.493(9)$ & C31-H31 & 0.95 \\
\hline 0.95 & $\mathrm{C} 32-\mathrm{C} 33$ & $1.389(10)$ \\
\hline $1.385(10)$ & C32-H32 & 0.95 \\
\hline 0.95 & $\mathrm{C} 33-\mathrm{C} 33 \mathrm{a}$ & $1.476(10)$ \\
\hline $1.480(9)$ & С33-Н33 & 0.95 \\
\hline 0.95 & $\mathrm{C} 33 \mathrm{a}-\mathrm{C} 34$ & $1.351(10)$ \\
\hline $1.365(9)$ & $\mathrm{C} 33 \mathrm{a}-\mathrm{C} 37 \mathrm{a}$ & $1.418(9)$ \\
\hline $1.423(9)$ & $\mathrm{C} 34-\mathrm{C} 35$ & $1.397(12)$ \\
\hline $1.375(10)$ & С $34-$ H34 & 0.95 \\
\hline 0.95 & $\mathrm{C} 35-\mathrm{C} 36$ & 1.367 (13) \\
\hline $1.405(10)$ & C35-H35 & 0.95 \\
\hline 0.95 & $\mathrm{C} 36-\mathrm{C} 37$ & $1.397(11)$ \\
\hline $1.386(9)$ & С $36-$ - 36 & 0.95 \\
\hline 0.95 & $\mathrm{C} 37-\mathrm{C} 37 \mathrm{a}$ & $1.383(10)$ \\
\hline $1.376(9)$ & C37-H37 & 0.95 \\
\hline 0.95 & $\mathrm{Pd}-\mathrm{C} 2$ & $2.148(7)$ \\
\hline $2.130(7)$ & $\mathrm{Pd}-\mathrm{C} 1$ & $2.158(7)$ \\
\hline $2.176(7)$ & $\mathrm{Pd}-\mathrm{C} 3$ & $2.202(6)$ \\
\hline $2.213(7)$ & $\mathrm{Pd}-\mathrm{Cl}^{\mathrm{iv}}$ & $2.3913(16)$ \\
\hline $2.3969(17)$ & $\mathrm{Pd}-\mathrm{Cl}$ & $2.4122(16)$ \\
\hline $2.4040(17)$ & $\mathrm{Pd}-\mathrm{C} 7 \mathrm{a}$ & $2.625(6)$ \\
\hline $2.4040(17)$ & $\mathrm{Pd}-\mathrm{C} 3 \mathrm{a}$ & $2.645(6)$ \\
\hline $1.427(9)$ & $\mathrm{Cl}-\mathrm{Pd}^{\mathrm{iv}}$ & $2.3913(16)$ \\
\hline $1.509(9)$ & $\mathrm{C} 1-\mathrm{C} 2$ & $1.416(10)$ \\
\hline 0.95 & $\mathrm{C} 1-\mathrm{C} 7 \mathrm{a}$ & $1.501(9)$ \\
\hline $1.414(10)$ & $\mathrm{C} 1-\mathrm{H} 1$ & 0.95 \\
\hline 0.95 & $\mathrm{C} 2-\mathrm{C} 3$ & $1.414(9)$ \\
\hline $1.468(8)$ & $\mathrm{C} 2-\mathrm{H} 2$ & 0.95 \\
\hline 0.95 & $\mathrm{C} 3-\mathrm{C} 3 \mathrm{a}$ & $1.471(9)$ \\
\hline $1.391(9)$ & $\mathrm{C} 3-\mathrm{H} 3$ & 0.95 \\
\hline $1.400(9)$ & $\mathrm{C} 3 \mathrm{a}-\mathrm{C} 4$ & $1.390(8)$ \\
\hline $1.401(9)$ & $\mathrm{C} 3 \mathrm{a}-\mathrm{C} 7 \mathrm{a}$ & $1.436(9)$ \\
\hline 0.95 & $\mathrm{C} 4-\mathrm{C} 5$ & 1.395 (11) \\
\hline $1.394(9)$ & $\mathrm{C} 4-\mathrm{H} 4$ & 0.95 \\
\hline 0.95 & $\mathrm{C} 5-\mathrm{C} 6$ & $1.396(11)$ \\
\hline $1.374(10)$ & $\mathrm{C} 5-\mathrm{H} 5$ & 0.95 \\
\hline 0.95 & $\mathrm{C} 6-\mathrm{C} 7$ & $1.393(10)$ \\
\hline $1.380(9)$ & $\mathrm{C} 6-\mathrm{H} 6$ & 0.95 \\
\hline 0.95 & $\mathrm{C} 7-\mathrm{C} 7 \mathrm{a}$ & $1.361(10)$ \\
\hline $2.142(6)$ & $\mathrm{C} 7-\mathrm{H} 7$ & 0.95 \\
\hline \multicolumn{3}{|l|}{$2.154(7)$} \\
\hline $37.6(3)$ & $\mathrm{C} 13-\mathrm{PD} 1-\mathrm{CL}^{\mathrm{i}}$ & $165.89(18)$ \\
\hline $39.1(3)$ & $\mathrm{C} 11-\mathrm{PD} 1-\mathrm{CL}^{\mathrm{i}}$ & $103.80(19)$ \\
\hline $62.4(3)$ & $\mathrm{CL} 1-\mathrm{PD} 1-\mathrm{CL}^{\mathrm{i}}$ & $87.36(6)$ \\
\hline $135.6(2)$ & $\mathrm{PD} 1-\mathrm{CL} 1-\mathrm{PD}^{\mathrm{i}}$ & $92.64(6)$ \\
\hline $105.75(18)$ & $\mathrm{C} 12-\mathrm{C} 11-\mathrm{C} 17 \mathrm{~A}$ & $108.0(6)$ \\
\hline $165.54(18)$ & $\mathrm{C} 12-\mathrm{C} 11-\mathrm{PD} 1$ & $68.0(4)$ \\
\hline $132.9(2)$ & $\mathrm{C} 17 \mathrm{~A}-\mathrm{C} 11-\mathrm{PD} 1$ & $89.9(4)$ \\
\hline
\end{tabular}




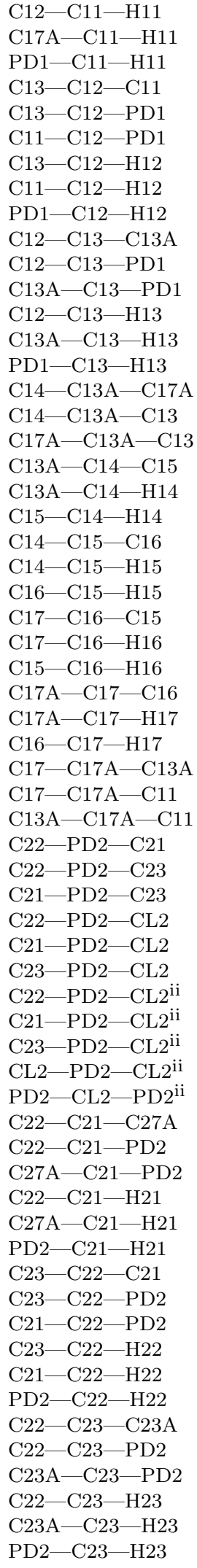

126
126
108.7
$106.1(6)$
$73.0(4)$
$72.9(4)$
127
127
119.2
$110.2(6)$
$69.5(4)$
$91.2(4)$
124.9
124.9
106.8
$119.5(6)$
$133.9(6)$
$106.5(6)$
$119.4(6)$
120.3
120.3
$121.2(7)$
119.4
119.4
$120.1(7)$
119.9
119.9
$118.2(6)$
120.9
120.9
$121.5(6)$
$132.2(6)$
$106.4(6)$
$38.7(3)$
$37.9(3)$
$63.1(3)$
$138.4(2)$
$166.54(19)$
$107.34(19)$
$130.4(2)$
126
$101.7(2)$
107.9
$164.65(19)$
$87.37(6)$
$92.63(6)$
$105.8(6)$
$68.9(4)$
$90.3(4)$
127.1
126.1
127.1
107.1
$107.8(6)$

719.2

$120.7(6)$ $131.8(6)$ $107.5(6)$ $118.0(6)$ 121 121 $120.4(6)$ 119.8 119.8 121.3 (6)

119.4

119.4

$118.8(7)$ 120.6

120.6 120.9 (6) $131.6(7)$ $107.5(6)$ 37.7 (3) 38.1 (2) 61.7 (3) $131.6(2)$ 102.7 (2) 163.63 (19) 138.0 (2) $167.2(2)$ 107.95 (19) 86.77 (6) 57.9 (2) 34.1 (2) $56.4(2)$ $108.14(16)$ 134.75 (17) 93.23 (6) 109.1 (6) 68.4 (4) 89.8 (4) 125.5

125.5

108.7 105.6 (6) 71.6 (4) 73.5 (4) 127.2

127.2

119.7 $110.2(6)$ 70.7 (4) 90.9 (4) 124.9 124.9 106 120.1 (8) 133.6 (8) 106.3 (6) $133.2(5)$ 75.4 (4) 55.0 (3) 119.1 (8) 120.5 120.5 120.8 (8) 


\begin{tabular}{|c|c|}
\hline C36-C35-H35 & 119.6 \\
\hline C34-C35-H35 & 119.6 \\
\hline $\mathrm{C} 35-\mathrm{C} 36-\mathrm{C} 37$ & $121.7(8)$ \\
\hline C35-C36-H36 & 119.1 \\
\hline C37-C36-H36 & 119.1 \\
\hline $\mathrm{C} 37 \mathrm{~A}-\mathrm{C} 37-\mathrm{C} 36$ & $116.8(8)$ \\
\hline $\mathrm{C} 37 \mathrm{~A}-\mathrm{C} 37-\mathrm{H} 37$ & 121.6 \\
\hline $\mathrm{C} 36-\mathrm{C} 37-\mathrm{H} 37$ & 121.6 \\
\hline $\mathrm{C} 37-\mathrm{C} 37 \mathrm{~A}-\mathrm{C} 33 \mathrm{~A}$ & $121.4(7)$ \\
\hline $\mathrm{C} 37-\mathrm{C} 37 \mathrm{~A}-\mathrm{C} 31$ & $132.7(7)$ \\
\hline $\mathrm{C} 33 \mathrm{~A}-\mathrm{C} 37 \mathrm{~A}-\mathrm{C} 31$ & $105.9(6)$ \\
\hline $\mathrm{C} 2-\mathrm{PD}-\mathrm{C} 1$ & $38.4(3)$ \\
\hline $\mathrm{C} 2-\mathrm{PD}-\mathrm{C} 3$ & $37.9(2)$ \\
\hline $\mathrm{C} 1-\mathrm{PD}-\mathrm{C} 3$ & $62.6(3)$ \\
\hline $\mathrm{C} 2-\mathrm{PD}-\mathrm{CL}^{\mathrm{iv}}$ & $132.20(19)$ \\
\hline $\mathrm{C} 1-\mathrm{PD}-\mathrm{CL}^{\mathrm{iv}}$ & $102.0(2)$ \\
\hline $\mathrm{C} 3-\mathrm{PD}-\mathrm{CL}^{\mathrm{iv}}$ & $163.63(18)$ \\
\hline $\mathrm{C} 2-\mathrm{PD}-\mathrm{CL}$ & $137.6(2)$ \\
\hline $\mathrm{C} 1-\mathrm{PD}-\mathrm{CL}$ & $167.82(18)$ \\
\hline $\mathrm{C} 3-\mathrm{PD}-\mathrm{CL}$ & $107.72(17)$ \\
\hline $\mathrm{CL}^{\mathrm{iv}}-\mathrm{PD}-\mathrm{CL}$ & $86.74(5)$ \\
\hline $\mathrm{C} 2-\mathrm{PD}-\mathrm{C} 7 \mathrm{~A}$ & $58.6(2)$ \\
\hline $\mathrm{C} 1-\mathrm{PD}-\mathrm{C} 7 \mathrm{~A}$ & $34.9(2)$ \\
\hline $\mathrm{C} 3-\mathrm{PD}-\mathrm{C} 7 \mathrm{~A}$ & $57.1(2)$ \\
\hline $\mathrm{CL}^{\mathrm{iv}}-\mathrm{PD}-\mathrm{C} 7 \mathrm{~A}$ & $107.31(16)$ \\
\hline $\mathrm{CL}-\mathrm{PD}-\mathrm{C} 7 \mathrm{~A}$ & $134.45(15)$ \\
\hline $\mathrm{C} 2-\mathrm{PD}-\mathrm{C} 3 \mathrm{~A}$ & $57.7(2)$ \\
\hline $\mathrm{C} 1-\mathrm{PD}-\mathrm{C} 3 \mathrm{~A}$ & $57.1(2)$ \\
\hline $\mathrm{C} 3-\mathrm{PD}-\mathrm{C} 3 \mathrm{~A}$ & $33.8(2)$ \\
\hline $\mathrm{CL}^{\mathrm{iv}}-\mathrm{PD}-\mathrm{C} 3 \mathrm{~A}$ & $134.20(15)$ \\
\hline $\mathrm{CL}-\mathrm{PD}-\mathrm{C} 3 \mathrm{~A}$ & $110.72(14)$ \\
\hline $\mathrm{C} 7 \mathrm{~A}-\mathrm{PD}-\mathrm{C} 3 \mathrm{~A}$ & $31.62(19)$ \\
\hline $\mathrm{PD}^{\mathrm{iv}}-\mathrm{CL}-\mathrm{PD}$ & $93.26(5)$ \\
\hline $\mathrm{C} 2-\mathrm{C} 1-\mathrm{C} 7 \mathrm{~A}$ & $108.9(6)$ \\
\hline $\mathrm{C} 2-\mathrm{C} 1-\mathrm{PD}$ & $70.4(4)$ \\
\hline $\mathrm{C} 7 \mathrm{~A}-\mathrm{C} 1-\mathrm{PD}$ & $89.9(4)$ \\
\hline $\mathrm{C} 2-\mathrm{C} 1-\mathrm{H} 1$ & 125.6 \\
\hline $\mathrm{C} 7 \mathrm{~A}-\mathrm{C} 1-\mathrm{H} 1$ & 125.6 \\
\hline $\mathrm{C} 12-\mathrm{PD} 1-\mathrm{CL} 1-\mathrm{PD} 1^{\mathrm{i}}$ & $-158.0(3)$ \\
\hline $\mathrm{C} 13-\mathrm{PD} 1-\mathrm{CL} 1-\mathrm{PD} 1^{\mathrm{i}}$ & $174.71(19)$ \\
\hline $\mathrm{C} 11-\mathrm{PD} 1-\mathrm{CL} 1-\mathrm{PD} 1^{\mathrm{i}}$ & $141.0(8)$ \\
\hline $\mathrm{CL} 1^{\mathrm{i}}-\mathrm{PD} 1-\mathrm{CL} 1-\mathrm{PD} 1^{\mathrm{i}}$ & 0 \\
\hline $\mathrm{C} 13-\mathrm{PD} 1-\mathrm{C} 11-\mathrm{C} 12$ & $39.1(4)$ \\
\hline $\mathrm{CL} 1-\mathrm{PD} 1-\mathrm{C} 11-\mathrm{C} 12$ & $76.2(9)$ \\
\hline $\mathrm{CL} 1{ }^{\mathrm{i}}-\mathrm{PD} 1-\mathrm{C} 11-\mathrm{C} 12$ & $-144.1(4)$ \\
\hline $\mathrm{C} 12-\mathrm{PD} 1-\mathrm{C} 11-\mathrm{C} 17 \mathrm{~A}$ & $-109.5(6)$ \\
\hline $\mathrm{C} 13-\mathrm{PD} 1-\mathrm{C} 11-\mathrm{C} 17 \mathrm{~A}$ & $-70.4(4)$ \\
\hline $\mathrm{CL} 1{ }^{\mathrm{i}}-\mathrm{PD} 1-\mathrm{C} 11-\mathrm{C} 17 \mathrm{~A}$ & $106.4(4)$ \\
\hline $\mathrm{C} 17 \mathrm{~A}-\mathrm{C} 11-\mathrm{C} 12-\mathrm{C} 13$ & $16.3(7)$ \\
\hline $\mathrm{PD} 1-\mathrm{C} 11-\mathrm{C} 12-\mathrm{C} 13$ & $-66.0(5)$ \\
\hline $\mathrm{C} 17 \mathrm{~A}-\mathrm{C} 11-\mathrm{C} 12-\mathrm{PD} 1$ & $82.3(4)$ \\
\hline $\mathrm{C} 11-\mathrm{PD} 1-\mathrm{C} 12-\mathrm{C} 13$ & $113.4(6)$ \\
\hline $\mathrm{CL} 1-\mathrm{PD} 1-\mathrm{C} 12-\mathrm{C} 13$ & $-46.4(5)$ \\
\hline $\mathrm{CL} 1{ }^{\mathrm{i}}-\mathrm{PD} 1-\mathrm{C} 12-\mathrm{C} 13$ & $164.4(3)$ \\
\hline $\mathrm{C} 13-\mathrm{PD} 1-\mathrm{C} 12-\mathrm{C} 11$ & $-113.4(6)$ \\
\hline $\mathrm{CL} 1-\mathrm{PD} 1-\mathrm{C} 12-\mathrm{C} 11$ & $-159.7(3)$ \\
\hline $\mathrm{CL} 11^{\mathrm{i}}-\mathrm{PD} 1-\mathrm{C} 12-\mathrm{C} 11$ & $51.0(5)$ \\
\hline $\mathrm{C} 11-\mathrm{C} 12-\mathrm{C} 13-\mathrm{C} 13 \mathrm{~A}$ & $-17.5(7)$ \\
\hline $\mathrm{PD} 1-\mathrm{C} 12-\mathrm{C} 13-\mathrm{C} 13 \mathrm{~A}$ & $-83.4(5)$ \\
\hline $\mathrm{C} 11-\mathrm{C} 12-\mathrm{C} 13-\mathrm{PD} 1$ & $66.0(4)$ \\
\hline $\mathrm{C} 11-\mathrm{PD} 1-\mathrm{C} 13-\mathrm{C} 12$ & $-40.8(4)$ \\
\hline $\mathrm{CL} 1-\mathrm{PD} 1-\mathrm{C} 13-\mathrm{C} 12$ & $148.2(4)$ \\
\hline
\end{tabular}

\begin{tabular}{|c|c|}
\hline $\mathrm{PD}-\mathrm{C} 1-\mathrm{H} 1$ & 106.9 \\
\hline $\mathrm{C} 3-\mathrm{C} 2-\mathrm{C} 1$ & $106.2(6)$ \\
\hline $\mathrm{C} 3-\mathrm{C} 2-\mathrm{PD}$ & $73.1(4)$ \\
\hline $\mathrm{C} 1-\mathrm{C} 2-\mathrm{PD}$ & $71.2(4)$ \\
\hline $\mathrm{C} 3-\mathrm{C} 2-\mathrm{H} 2$ & 126.9 \\
\hline $\mathrm{C} 1-\mathrm{C} 2-\mathrm{H} 2$ & 126.9 \\
\hline $\mathrm{PD}-\mathrm{C} 2-\mathrm{H} 2$ & 120.7 \\
\hline $\mathrm{C} 2-\mathrm{C} 3-\mathrm{C} 3 \mathrm{~A}$ & $109.2(6)$ \\
\hline $\mathrm{C} 2-\mathrm{C} 3-\mathrm{PD}$ & $69.0(4)$ \\
\hline $\mathrm{C} 3 \mathrm{~A}-\mathrm{C} 3-\mathrm{PD}$ & $89.9(4)$ \\
\hline $\mathrm{C} 2-\mathrm{C} 3-\mathrm{H} 3$ & 125.4 \\
\hline $\mathrm{C} 3 \mathrm{~A}-\mathrm{C} 3-\mathrm{H} 3$ & 125.4 \\
\hline $\mathrm{PD}-\mathrm{C} 3-\mathrm{H} 3$ & 108.2 \\
\hline $\mathrm{C} 4-\mathrm{C} 3 \mathrm{~A}-\mathrm{C} 7 \mathrm{~A}$ & $119.9(6)$ \\
\hline $\mathrm{C} 4-\mathrm{C} 3 \mathrm{~A}-\mathrm{C} 3$ & $133.0(6)$ \\
\hline $\mathrm{C} 7 \mathrm{~A}-\mathrm{C} 3 \mathrm{~A}-\mathrm{C} 3$ & $107.0(5)$ \\
\hline $\mathrm{C} 4-\mathrm{C} 3 \mathrm{~A}-\mathrm{PD}$ & $134.5(5)$ \\
\hline $\mathrm{C} 7 \mathrm{~A}-\mathrm{C} 3 \mathrm{~A}-\mathrm{PD}$ & $73.4(4)$ \\
\hline $\mathrm{C} 3-\mathrm{C} 3 \mathrm{~A}-\mathrm{PD}$ & $56.3(3)$ \\
\hline $\mathrm{C} 3 \mathrm{~A}-\mathrm{C} 4-\mathrm{C} 5$ & $117.6(7)$ \\
\hline $\mathrm{C} 3 \mathrm{~A}-\mathrm{C} 4-\mathrm{H} 4$ & 121.2 \\
\hline $\mathrm{C} 5-\mathrm{C} 4-\mathrm{H} 4$ & 121.2 \\
\hline $\mathrm{C} 4-\mathrm{C} 5-\mathrm{C} 6$ & $121.8(7)$ \\
\hline $\mathrm{C} 4-\mathrm{C} 5-\mathrm{H} 5$ & 119.1 \\
\hline $\mathrm{C} 6-\mathrm{C} 5-\mathrm{H} 5$ & 119.1 \\
\hline $\mathrm{C} 7-\mathrm{C} 6-\mathrm{C} 5$ & $120.8(7)$ \\
\hline $\mathrm{C} 7-\mathrm{C} 6-\mathrm{H} 6$ & 119.6 \\
\hline $\mathrm{C} 5-\mathrm{C} 6-\mathrm{H} 6$ & 119.6 \\
\hline $\mathrm{C} 7 \mathrm{~A}-\mathrm{C} 7-\mathrm{C} 6$ & $118.3(7)$ \\
\hline $\mathrm{C} 7 \mathrm{~A}-\mathrm{C} 7-\mathrm{H} 7$ & 120.9 \\
\hline $\mathrm{C} 6-\mathrm{C} 7-\mathrm{H} 7$ & 120.9 \\
\hline $\mathrm{C} 7-\mathrm{C} 7 \mathrm{~A}-\mathrm{C} 3 \mathrm{~A}$ & $121.6(6)$ \\
\hline $\mathrm{C} 7-\mathrm{C} 7 \mathrm{~A}-\mathrm{C} 1$ & $133.0(7)$ \\
\hline $\mathrm{C} 3 \mathrm{~A}-\mathrm{C} 7 \mathrm{~A}-\mathrm{C} 1$ & $105.3(6)$ \\
\hline $\mathrm{C} 7-\mathrm{C} 7 \mathrm{~A}-\mathrm{PD}$ & $132.8(5)$ \\
\hline $\mathrm{C} 3 \mathrm{~A}-\mathrm{C} 7 \mathrm{~A}-\mathrm{PD}$ & $74.9(4)$ \\
\hline $\mathrm{C} 1-\mathrm{C} 7 \mathrm{~A}-\mathrm{PD}$ & $55.3(3)$ \\
\hline $\mathrm{CL} 1{ }^{\mathrm{i}}-\mathrm{PD} 1-\mathrm{C} 13-\mathrm{C} 12$ & $-54.0(9)$ \\
\hline $\mathrm{C} 12-\mathrm{PD} 1-\mathrm{C} 13-\mathrm{C} 13 \mathrm{~A}$ & $111.2(6)$ \\
\hline $\mathrm{C} 11-\mathrm{PD} 1-\mathrm{C} 13-\mathrm{C} 13 \mathrm{~A}$ & $70.4(4)$ \\
\hline $\mathrm{CL} 1-\mathrm{PD} 1-\mathrm{C} 13-\mathrm{C} 13 \mathrm{~A}$ & $-100.6(4)$ \\
\hline $\mathrm{CL} 1{ }^{\mathrm{i}}-\mathrm{PD} 1-\mathrm{C} 13-\mathrm{C} 13 \mathrm{~A}$ & $57.2(9)$ \\
\hline $\mathrm{C} 12-\mathrm{C} 13-\mathrm{C} 13 \mathrm{~A}-\mathrm{C} 14$ & $-171.2(7)$ \\
\hline $\mathrm{PD} 1-\mathrm{C} 13-\mathrm{C} 13 \mathrm{~A}-\mathrm{C} 14$ & $120.3(8)$ \\
\hline $\mathrm{C} 12-\mathrm{C} 13-\mathrm{C} 13 \mathrm{~A}-\mathrm{C} 17 \mathrm{~A}$ & $11.9(7)$ \\
\hline $\mathrm{PD} 1-\mathrm{C} 13-\mathrm{C} 13 \mathrm{~A}-\mathrm{C} 17 \mathrm{~A}$ & $-56.6(5)$ \\
\hline $\mathrm{C} 13-\mathrm{C} 13 \mathrm{~A}-\mathrm{C} 14-\mathrm{C} 15$ & $-177.8(7)$ \\
\hline $\mathrm{C} 16-\mathrm{C} 17-\mathrm{C} 17 \mathrm{~A}-\mathrm{C} 13 \mathrm{~A}$ & $1.0(9)$ \\
\hline $\mathrm{C} 16-\mathrm{C} 17-\mathrm{C} 17 \mathrm{~A}-\mathrm{C} 11$ & $-179.3(6)$ \\
\hline $\mathrm{C} 14-\mathrm{C} 13 \mathrm{~A}-\mathrm{C} 17 \mathrm{~A}-\mathrm{C} 17$ & $1.0(9)$ \\
\hline $\mathrm{C} 13-\mathrm{C} 13 \mathrm{~A}-\mathrm{C} 17 \mathrm{~A}-\mathrm{C} 17$ & $178.4(6)$ \\
\hline $\mathrm{C} 14-\mathrm{C} 13 \mathrm{~A}-\mathrm{C} 17 \mathrm{~A}-\mathrm{C} 11$ & $-178.8(6)$ \\
\hline $\mathrm{C} 13-\mathrm{C} 13 \mathrm{~A}-\mathrm{C} 17 \mathrm{~A}-\mathrm{C} 11$ & $-1.3(7)$ \\
\hline $\mathrm{C} 12-\mathrm{C} 11-\mathrm{C} 17 \mathrm{~A}-\mathrm{C} 17$ & $171.2(7)$ \\
\hline $\mathrm{PD} 1-\mathrm{C} 11-\mathrm{C} 17 \mathrm{~A}-\mathrm{C} 17$ & $-122.1(7)$ \\
\hline $\mathrm{C} 12-\mathrm{C} 11-\mathrm{C} 17 \mathrm{~A}-\mathrm{C} 13 \mathrm{~A}$ & $-9.1(7)$ \\
\hline $\mathrm{PD} 1-\mathrm{C} 11-\mathrm{C} 17 \mathrm{~A}-\mathrm{C} 13 \mathrm{~A}$ & $57.7(5)$ \\
\hline $\mathrm{C} 22-\mathrm{PD} 2-\mathrm{CL} 2-\mathrm{PD} 2^{\mathrm{ii}}$ & $157.7(3)$ \\
\hline $\mathrm{C} 21-\mathrm{PD} 2-\mathrm{CL} 2-\mathrm{PD} 2^{\mathrm{ii}}$ & $-132.6(8)$ \\
\hline $\mathrm{C} 23-\mathrm{PD} 2-\mathrm{CL} 2-\mathrm{PD} 2^{\mathrm{ii}}$ & $-175.51(17)$ \\
\hline $\mathrm{CL} 22^{\mathrm{ii}}-\mathrm{PD} 2-\mathrm{CL} 2-\mathrm{PD} 2^{\mathrm{ii}}$ & 0 \\
\hline
\end{tabular}




\begin{tabular}{|c|c|}
\hline $\mathrm{C} 23-\mathrm{PD} 2-\mathrm{C} 21-\mathrm{C} 22$ & $-38.7(4)$ \\
\hline $\mathrm{CL} 2-\mathrm{PD} 2-\mathrm{C} 21-\mathrm{C} 22$ & $-85.5(9)$ \\
\hline $\mathrm{CL} 22^{\mathrm{ii}}-\mathrm{PD} 2-\mathrm{C} 21-\mathrm{C} 22$ & $143.2(4)$ \\
\hline $\mathrm{C} 22-\mathrm{PD} 2-\mathrm{C} 21-\mathrm{C} 27 \mathrm{~A}$ & $106.8(6)$ \\
\hline $\mathrm{C} 23-\mathrm{PD} 2-\mathrm{C} 21-\mathrm{C} 27 \mathrm{~A}$ & $68.2(4)$ \\
\hline $\mathrm{CL} 22^{\mathrm{ii}}-\mathrm{PD} 2-\mathrm{C} 21-\mathrm{C} 27 \mathrm{~A}$ & $-110.0(4)$ \\
\hline $\mathrm{C} 27 \mathrm{~A}-\mathrm{C} 21-\mathrm{C} 22-\mathrm{C} 23$ & $-17.8(8)$ \\
\hline $\mathrm{PD} 2-\mathrm{C} 21-\mathrm{C} 22-\mathrm{C} 23$ & $66.3(5)$ \\
\hline $\mathrm{C} 27 \mathrm{~A}-\mathrm{C} 21-\mathrm{C} 22-\mathrm{PD} 2$ & $-84.1(5)$ \\
\hline $\mathrm{C} 21-\mathrm{PD} 2-\mathrm{C} 22-\mathrm{C} 23$ & $-115.1(6)$ \\
\hline $\mathrm{CL} 2-\mathrm{PD} 2-\mathrm{C} 22-\mathrm{C} 23$ & $44.5(5)$ \\
\hline $\mathrm{CL} 22^{\mathrm{ii}}-\mathrm{PD} 2-\mathrm{C} 22-\mathrm{C} 23$ & $-165.4(3)$ \\
\hline $\mathrm{C} 23-\mathrm{PD} 2-\mathrm{C} 22-\mathrm{C} 21$ & $115.1(6)$ \\
\hline $\mathrm{CL} 2-\mathrm{PD} 2-\mathrm{C} 22-\mathrm{C} 21$ & $159.6(3)$ \\
\hline $\mathrm{CL} 22^{\mathrm{ii}}-\mathrm{PD} 2-\mathrm{C} 22-\mathrm{C} 21$ & $-50.4(5)$ \\
\hline $\mathrm{C} 21-\mathrm{C} 22-\mathrm{C} 23-\mathrm{C} 23 \mathrm{~A}$ & $18.3(8)$ \\
\hline $\mathrm{PD} 2-\mathrm{C} 22-\mathrm{C} 23-\mathrm{C} 23 \mathrm{~A}$ & $83.4(4)$ \\
\hline $\mathrm{C} 21-\mathrm{C} 22-\mathrm{C} 23-\mathrm{PD} 2$ & $-65.1(5)$ \\
\hline $\mathrm{C} 21-\mathrm{PD} 2-\mathrm{C} 23-\mathrm{C} 22$ & $39.4(4)$ \\
\hline $\mathrm{CL} 2-\mathrm{PD} 2-\mathrm{C} 23-\mathrm{C} 22$ & $-150.8(4)$ \\
\hline $\mathrm{CL} 22^{\mathrm{ii}}-\mathrm{PD} 2-\mathrm{C} 23-\mathrm{C} 22$ & $46.4(8)$ \\
\hline $\mathrm{C} 22-\mathrm{PD} 2-\mathrm{C} 23-\mathrm{C} 23 \mathrm{~A}$ & $-109.2(6)$ \\
\hline $\mathrm{C} 21-\mathrm{PD} 2-\mathrm{C} 23-\mathrm{C} 23 \mathrm{~A}$ & $-69.7(4)$ \\
\hline CL2-PD2-C23-C23A & $100.0(4)$ \\
\hline $\mathrm{CL} 2^{\mathrm{ii}}-\mathrm{PD} 2-\mathrm{C} 23-\mathrm{C} 23 \mathrm{~A}$ & $-62.8(8)$ \\
\hline $\mathrm{C} 22-\mathrm{C} 23-\mathrm{C} 23 \mathrm{~A}-\mathrm{C} 24$ & $170.1(7)$ \\
\hline $\mathrm{PD} 2-\mathrm{C} 23-\mathrm{C} 23 \mathrm{~A}-\mathrm{C} 24$ & $-122.9(7)$ \\
\hline $\mathrm{C} 22-\mathrm{C} 23-\mathrm{C} 23 \mathrm{~A}-\mathrm{C} 27 \mathrm{~A}$ & $-11.3(7)$ \\
\hline $\mathrm{PD} 2-\mathrm{C} 23-\mathrm{C} 23 \mathrm{~A}-\mathrm{C} 27 \mathrm{~A}$ & $55.6(5)$ \\
\hline $\mathrm{C} 23-\mathrm{C} 23 \mathrm{~A}-\mathrm{C} 24-\mathrm{C} 25$ & $178.7(6)$ \\
\hline $\mathrm{C} 26-\mathrm{C} 27-\mathrm{C} 27 \mathrm{~A}-\mathrm{C} 21$ & $-179.3(7)$ \\
\hline $\mathrm{C} 23-\mathrm{C} 23 \mathrm{~A}-\mathrm{C} 27 \mathrm{~A}-\mathrm{C} 27$ & $-177.8(6)$ \\
\hline $\mathrm{C} 24-\mathrm{C} 23 \mathrm{~A}-\mathrm{C} 27 \mathrm{~A}-\mathrm{C} 21$ & $178.9(6)$ \\
\hline $\mathrm{C} 23-\mathrm{C} 23 \mathrm{~A}-\mathrm{C} 27 \mathrm{~A}-\mathrm{C} 21$ & $0.2(7)$ \\
\hline $\mathrm{C} 22-\mathrm{C} 21-\mathrm{C} 27 \mathrm{~A}-\mathrm{C} 27$ & $-171.5(7)$ \\
\hline $\mathrm{PD} 2-\mathrm{C} 21-\mathrm{C} 27 \mathrm{~A}-\mathrm{C} 27$ & $120.3(7)$ \\
\hline $\mathrm{C} 22-\mathrm{C} 21-\mathrm{C} 27 \mathrm{~A}-\mathrm{C} 23 \mathrm{~A}$ & $10.8(8)$ \\
\hline $\mathrm{PD} 2-\mathrm{C} 21-\mathrm{C} 27 \mathrm{~A}-\mathrm{C} 23 \mathrm{~A}$ & $-57.4(5)$ \\
\hline $\mathrm{C} 32-\mathrm{PD} 3-\mathrm{CL} 3-\mathrm{PD} 3^{\mathrm{iii}}$ & $158.5(3)$ \\
\hline C33-PD3-CL3-PD3 ${ }^{\text {iii }}$ & $-138.2(9)$ \\
\hline C31-PD3-CL3-PD3 $3^{\text {iii }}$ & $-172.7(2)$ \\
\hline $\mathrm{CL} 3^{\mathrm{iii}}-\mathrm{PD} 3-\mathrm{CL} 3-\mathrm{PD} 3^{\mathrm{iii}}$ & 0 \\
\hline $\mathrm{C} 33 \mathrm{~A}-\mathrm{PD} 3-\mathrm{CL} 3-\mathrm{PD} 3^{\mathrm{iii}}$ & $-112.5(2)$ \\
\hline $\mathrm{C} 33-\mathrm{PD} 3-\mathrm{C} 31-\mathrm{C} 32$ & $39.7(4)$ \\
\hline $\mathrm{CL}^{\mathrm{iii}}-\mathrm{PD} 3-\mathrm{C} 31-\mathrm{C} 32$ & $58.4(9)$ \\
\hline CL3-PD3-C31-C32 & $-148.5(4)$ \\
\hline $\mathrm{C} 33 \mathrm{~A}-\mathrm{PD} 3-\mathrm{C} 31-\mathrm{C} 32$ & $79.2(5)$ \\
\hline C32-PD3-C31-C37A & $-110.6(6)$ \\
\hline $\mathrm{C} 33-\mathrm{PD} 3-\mathrm{C} 31-\mathrm{C} 37 \mathrm{~A}$ & $-70.9(4)$ \\
\hline $\mathrm{CL} 3^{\mathrm{iii}}-\mathrm{PD} 3-\mathrm{C} 31-\mathrm{C} 37 \mathrm{~A}$ & $-52.3(9)$ \\
\hline CL3-PD3-C31-C37A & $100.9(4)$ \\
\hline $\mathrm{C} 33 \mathrm{~A}-\mathrm{PD} 3-\mathrm{C} 31-\mathrm{C} 37 \mathrm{~A}$ & $-31.4(4)$ \\
\hline $\mathrm{C} 37 \mathrm{~A}-\mathrm{C} 31-\mathrm{C} 32-\mathrm{C} 33$ & $17.0(7)$ \\
\hline PD3-C31-C32-C33 & $-64.9(4)$ \\
\hline $\mathrm{C} 37 \mathrm{~A}-\mathrm{C} 31-\mathrm{C} 32-\mathrm{PD} 3$ & $81.9(4)$ \\
\hline $\mathrm{C} 31-\mathrm{PD} 3-\mathrm{C} 32-\mathrm{C} 33$ & $113.2(6)$ \\
\hline $\mathrm{CL}^{\mathrm{iii}}-\mathrm{PD} 3-\mathrm{C} 32-\mathrm{C} 33$ & $-48.1(5)$ \\
\hline CL3-PD3-C32-C33 & $161.2(3)$ \\
\hline $\mathrm{C} 33 \mathrm{~A}-\mathrm{PD} 3-\mathrm{C} 32-\mathrm{C} 33$ & $38.1(4)$ \\
\hline $\mathrm{C} 33-\mathrm{PD} 3-\mathrm{C} 32-\mathrm{C} 31$ & $-113.2(6)$ \\
\hline $\mathrm{CL}^{\mathrm{iii}}{ }^{-\mathrm{PD}} 3-\mathrm{C} 32-\mathrm{C} 31$ & $-161.3(3)$ \\
\hline CL3-PD3-C32-C31 & $48.0(5)$ \\
\hline
\end{tabular}

\begin{tabular}{|c|c|}
\hline $\mathrm{C} 33 \mathrm{~A}-\mathrm{PD} 3-\mathrm{C} 32-\mathrm{C} 31$ & $-75.0(4)$ \\
\hline $\mathrm{C} 31-\mathrm{C} 32-\mathrm{C} 33-\mathrm{C} 33 \mathrm{~A}$ & $-17.5(7)$ \\
\hline $\mathrm{PD} 3-\mathrm{C} 32-\mathrm{C} 33-\mathrm{C} 33 \mathrm{~A}$ & $-83.6(5)$ \\
\hline $\mathrm{C} 31-\mathrm{C} 32-\mathrm{C} 33-\mathrm{PD} 3$ & $66.2(4)$ \\
\hline $\mathrm{C} 31-\mathrm{PD} 3-\mathrm{C} 33-\mathrm{C} 32$ & $-40.1(4)$ \\
\hline $\mathrm{C} 33 \mathrm{~A}-\mathrm{PD} 3-\mathrm{C} 33-\mathrm{C} 32$ & $-111.1(6)$ \\
\hline $\mathrm{C} 32-\mathrm{PD} 3-\mathrm{C} 33-\mathrm{C} 33 \mathrm{~A}$ & $111.1(6)$ \\
\hline $\mathrm{C} 31-\mathrm{PD} 3-\mathrm{C} 33-\mathrm{C} 33 \mathrm{~A}$ & $71.0(4)$ \\
\hline $\mathrm{C} 32-\mathrm{C} 33-\mathrm{C} 33 \mathrm{~A}-\mathrm{C} 34$ & $-171.1(7)$ \\
\hline PD3-C33-C33A-C34 & $119.1(8)$ \\
\hline $\mathrm{C} 32-\mathrm{C} 33-\mathrm{C} 33 \mathrm{~A}-\mathrm{C} 37 \mathrm{~A}$ & $11.3(7)$ \\
\hline $\mathrm{PD} 3-\mathrm{C} 33-\mathrm{C} 33 \mathrm{~A}-\mathrm{C} 37 \mathrm{~A}$ & $-58.4(5)$ \\
\hline $\mathrm{C} 32-\mathrm{C} 33-\mathrm{C} 33 \mathrm{~A}-\mathrm{PD} 3$ & $69.7(5)$ \\
\hline $\mathrm{C} 32-\mathrm{PD} 3-\mathrm{C} 33 \mathrm{~A}-\mathrm{C} 34$ & $-162.1(9)$ \\
\hline $\mathrm{C} 31-\mathrm{PD} 3-\mathrm{C} 33 \mathrm{~A}-\mathrm{C} 34$ & $152.2(9)$ \\
\hline CL3 ${ }^{\mathrm{iii}}-\mathrm{PD} 3-\mathrm{C} 33 \mathrm{~A}-\mathrm{C} 34$ & $-33.8(9)$ \\
\hline CL3-PD3-C33A-C34 & $70.1(9)$ \\
\hline $\mathrm{C} 32-\mathrm{PD} 3-\mathrm{C} 33 \mathrm{~A}-\mathrm{C} 37 \mathrm{~A}$ & $80.0(4)$ \\
\hline $\mathrm{C} 33-\mathrm{PD} 3-\mathrm{C} 33 \mathrm{~A}-\mathrm{C} 37 \mathrm{~A}$ & $122.4(6)$ \\
\hline $\mathrm{C} 31-\mathrm{PD} 3-\mathrm{C} 33 \mathrm{~A}-\mathrm{C} 37 \mathrm{~A}$ & $34.3(4)$ \\
\hline 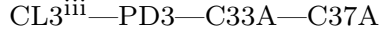 & $-151.7(4)$ \\
\hline $\mathrm{CL} 3-\mathrm{PD} 3-\mathrm{C} 33 \mathrm{~A}-\mathrm{C} 37 \mathrm{~A}$ & $-47.8(5)$ \\
\hline $\mathrm{C} 32-\mathrm{PD} 3-\mathrm{C} 33 \mathrm{~A}-\mathrm{C} 33$ & $-42.4(4)$ \\
\hline $\mathrm{C} 31-\mathrm{PD} 3-\mathrm{C} 33 \mathrm{~A}-\mathrm{C} 33$ & $-88.1(5)$ \\
\hline $\mathrm{CL} 3^{\mathrm{iii}}-\mathrm{PD} 3-\mathrm{C} 33 \mathrm{~A}-\mathrm{C} 33$ & $85.9(4)$ \\
\hline CL3-PD3-C33A-C33 & $-170.2(4)$ \\
\hline $\mathrm{C} 33-\mathrm{C} 33 \mathrm{~A}-\mathrm{C} 34-\mathrm{C} 35$ & $-178.4(7)$ \\
\hline PD3-C33A-C34-C35 & $-99.6(9)$ \\
\hline $\mathrm{C} 34-\mathrm{C} 35-\mathrm{C} 36-\mathrm{C} 37$ & $0.00(12)$ \\
\hline $\mathrm{C} 36-\mathrm{C} 37-\mathrm{C} 37 \mathrm{~A}-\mathrm{C} 31$ & $179.0(6)$ \\
\hline $\mathrm{C} 33-\mathrm{C} 33 \mathrm{~A}-\mathrm{C} 37 \mathrm{~A}-\mathrm{C} 37$ & $177.8(6)$ \\
\hline $\mathrm{PD} 3-\mathrm{C} 33 \mathrm{~A}-\mathrm{C} 37 \mathrm{~A}-\mathrm{C} 37$ & $131.7(6)$ \\
\hline $\mathrm{C} 34-\mathrm{C} 33 \mathrm{~A}-\mathrm{C} 37 \mathrm{~A}-\mathrm{C} 31$ & $-178.4(6)$ \\
\hline $\mathrm{C} 33-\mathrm{C} 33 \mathrm{~A}-\mathrm{C} 37 \mathrm{~A}-\mathrm{C} 31$ & $-0.5(7)$ \\
\hline $\mathrm{PD} 3-\mathrm{C} 33 \mathrm{~A}-\mathrm{C} 37 \mathrm{~A}-\mathrm{C} 31$ & $-46.6(4)$ \\
\hline $\mathrm{C} 32-\mathrm{C} 31-\mathrm{C} 37 \mathrm{~A}-\mathrm{C} 37$ & $171.8(7)$ \\
\hline $\mathrm{PD} 3-\mathrm{C} 31-\mathrm{C} 37 \mathrm{~A}-\mathrm{C} 37$ & $-121.1(7)$ \\
\hline $\mathrm{C} 32-\mathrm{C} 31-\mathrm{C} 37 \mathrm{~A}-\mathrm{C} 33 \mathrm{~A}$ & $-10.2(7)$ \\
\hline $\mathrm{PD} 3-\mathrm{C} 31-\mathrm{C} 37 \mathrm{~A}-\mathrm{C} 33 \mathrm{~A}$ & $56.9(5)$ \\
\hline $\mathrm{C} 2-\mathrm{PD}-\mathrm{CL}-\mathrm{PD}^{\mathrm{iv}}$ & $159.2(3)$ \\
\hline $\mathrm{C} 3-\mathrm{PD}-\mathrm{CL}-\mathrm{PD}^{\mathrm{iv}}$ & $-172.18(18)$ \\
\hline $\mathrm{CL}^{\mathrm{iv}}-\mathrm{PD}-\mathrm{CL}-\mathrm{PD}^{\mathrm{iv}}$ & 0 \\
\hline $\mathrm{C} 7 \mathrm{~A}-\mathrm{PD}-\mathrm{CL}-\mathrm{PD}^{\mathrm{iv}}$ & $-111.2(2)$ \\
\hline $\mathrm{C} 3 \mathrm{~A}-\mathrm{PD}-\mathrm{CL}-\mathrm{PD}^{\mathrm{iv}}$ & $-136.48(15)$ \\
\hline $\mathrm{C} 3-\mathrm{PD}-\mathrm{C} 1-\mathrm{C} 2$ & $-39.1(4)$ \\
\hline $\mathrm{CL}^{\mathrm{iv}}-\mathrm{PD}-\mathrm{C} 1-\mathrm{C} 2$ & $146.8(3)$ \\
\hline $\mathrm{C} 7 \mathrm{~A}-\mathrm{PD}-\mathrm{C} 1-\mathrm{C} 2$ & $-110.1(6)$ \\
\hline $\mathrm{C} 3 \mathrm{~A}-\mathrm{PD}-\mathrm{C} 1-\mathrm{C} 2$ & $-77.9(4)$ \\
\hline $\mathrm{C} 2-\mathrm{PD}-\mathrm{C} 1-\mathrm{C} 7 \mathrm{~A}$ & $110.1(6)$ \\
\hline $\mathrm{C} 3-\mathrm{PD}-\mathrm{C} 1-\mathrm{C} 7 \mathrm{~A}$ & $71.0(4)$ \\
\hline $\mathrm{C} 3 \mathrm{~A}-\mathrm{PD}-\mathrm{C} 1-\mathrm{C} 7 \mathrm{~A}$ & $32.2(4)$ \\
\hline $\mathrm{C} 7 \mathrm{~A}-\mathrm{C} 1-\mathrm{C} 2-\mathrm{C} 3$ & $-17.6(7)$ \\
\hline $\mathrm{PD}-\mathrm{C} 1-\mathrm{C} 2-\mathrm{C} 3$ & $65.2(4)$ \\
\hline $\mathrm{C} 7 \mathrm{~A}-\mathrm{C} 1-\mathrm{C} 2-\mathrm{PD}$ & $-82.8(5)$ \\
\hline $\mathrm{C} 1-\mathrm{PD}-\mathrm{C} 2-\mathrm{C} 3$ & $-114.3(6)$ \\
\hline $\mathrm{CL}^{\mathrm{iv}}-\mathrm{PD}-\mathrm{C} 2-\mathrm{C} 3$ & $-160.7(3)$ \\
\hline $\mathrm{CL}-\mathrm{PD}-\mathrm{C} 2-\mathrm{C} 3$ & $47.9(5)$ \\
\hline $\mathrm{C} 7 \mathrm{~A}-\mathrm{PD}-\mathrm{C} 2-\mathrm{C} 3$ & $-75.4(4)$ \\
\hline $\mathrm{C} 3 \mathrm{~A}-\mathrm{PD}-\mathrm{C} 2-\mathrm{C} 3$ & $-38.0(4)$ \\
\hline $\mathrm{C} 3-\mathrm{PD}-\mathrm{C} 2-\mathrm{C} 1$ & $114.3(6)$ \\
\hline $\mathrm{CL}^{\mathrm{iv}}-\mathrm{PD}-\mathrm{C} 2-\mathrm{C} 1$ & $-46.3(4)$ \\
\hline $\mathrm{CL}-\mathrm{PD}-\mathrm{C} 2-\mathrm{C} 1$ & $162.2(3)$ \\
\hline
\end{tabular}




\begin{tabular}{|c|c|c|c|}
\hline $\mathrm{C} 7 \mathrm{~A}-\mathrm{PD}-\mathrm{C} 2-\mathrm{C} 1$ & $38.9(4)$ & $\mathrm{CL}-\mathrm{PD}-\mathrm{C} 3 \mathrm{~A}-\mathrm{C} 3$ & $-91.1(4)$ \\
\hline $\mathrm{C} 3 \mathrm{~A}-\mathrm{PD}-\mathrm{C} 2-\mathrm{C} 1$ & $76.3(4)$ & $\mathrm{C} 7 \mathrm{~A}-\mathrm{PD}-\mathrm{C} 3 \mathrm{~A}-\mathrm{C} 3$ & $124.4(6)$ \\
\hline $\mathrm{C} 1-\mathrm{C} 2-\mathrm{C} 3-\mathrm{C} 3 \mathrm{~A}$ & $18.2(7)$ & $\mathrm{C} 3-\mathrm{C} 3 \mathrm{~A}-\mathrm{C} 4-\mathrm{C} 5$ & $178.4(7)$ \\
\hline $\mathrm{PD}-\mathrm{C} 2-\mathrm{C} 3-\mathrm{C} 3 \mathrm{~A}$ & $82.1(5)$ & $\mathrm{PD}-\mathrm{C} 3 \mathrm{~A}-\mathrm{C} 4-\mathrm{C} 5$ & $96.7(8)$ \\
\hline $\mathrm{C} 1-\mathrm{C} 2-\mathrm{C} 3-\mathrm{PD}$ & $-63.9(4)$ & $\mathrm{C} 6-\mathrm{C} 7-\mathrm{C} 7 \mathrm{~A}-\mathrm{C} 1$ & $-179.0(7)$ \\
\hline $\mathrm{C} 1-\mathrm{PD}-\mathrm{C} 3-\mathrm{C} 2$ & $39.6(4)$ & $\mathrm{C} 6-\mathrm{C} 7-\mathrm{C} 7 \mathrm{~A}-\mathrm{PD}$ & $-100.4(8)$ \\
\hline $\mathrm{CL}^{\mathrm{iv}}-\mathrm{PD}-\mathrm{C} 3-\mathrm{C} 2$ & $60.5(8)$ & $\mathrm{C} 3-\mathrm{C} 3 \mathrm{~A}-\mathrm{C} 7 \mathrm{~A}-\mathrm{C} 7$ & $-177.2(6)$ \\
\hline $\mathrm{CL}-\mathrm{PD}-\mathrm{C} 3-\mathrm{C} 2$ & $-148.3(4)$ & $\mathrm{PD}-\mathrm{C} 3 \mathrm{~A}-\mathrm{C} 7 \mathrm{~A}-\mathrm{C} 7$ & $-131.3(7)$ \\
\hline $\mathrm{C} 7 \mathrm{~A}-\mathrm{PD}-\mathrm{C} 3-\mathrm{C} 2$ & $79.7(4)$ & $\mathrm{C} 4-\mathrm{C} 3 \mathrm{~A}-\mathrm{C} 7 \mathrm{~A}-\mathrm{C} 1$ & $179.0(6)$ \\
\hline $\mathrm{C} 3 \mathrm{~A}-\mathrm{PD}-\mathrm{C} 3-\mathrm{C} 2$ & $110.7(6)$ & $\mathrm{C} 3-\mathrm{C} 3 \mathrm{~A}-\mathrm{C} 7 \mathrm{~A}-\mathrm{C} 1$ & $0.8(7)$ \\
\hline $\mathrm{C} 2-\mathrm{PD}-\mathrm{C} 3-\mathrm{C} 3 \mathrm{~A}$ & $-110.7(6)$ & $\mathrm{PD}-\mathrm{C} 3 \mathrm{~A}-\mathrm{C} 7 \mathrm{~A}-\mathrm{C} 1$ & $46.7(4)$ \\
\hline $\mathrm{C} 1-\mathrm{PD}-\mathrm{C} 3-\mathrm{C} 3 \mathrm{~A}$ & $-71.1(4)$ & $\mathrm{C} 4-\mathrm{C} 3 \mathrm{~A}-\mathrm{C} 7 \mathrm{~A}-\mathrm{PD}$ & $132.3(6)$ \\
\hline $\mathrm{CL}^{\mathrm{iv}}-\mathrm{PD}-\mathrm{C} 3-\mathrm{C} 3 \mathrm{~A}$ & $-50.2(8)$ & $\mathrm{C} 3-\mathrm{C} 3 \mathrm{~A}-\mathrm{C} 7 \mathrm{~A}-\mathrm{PD}$ & $-45.9(4)$ \\
\hline $\mathrm{CL}-\mathrm{PD}-\mathrm{C} 3-\mathrm{C} 3 \mathrm{~A}$ & $101.0(4)$ & $\mathrm{C} 2-\mathrm{C} 1-\mathrm{C} 7 \mathrm{~A}-\mathrm{C} 7$ & $-171.9(8)$ \\
\hline $\mathrm{C} 7 \mathrm{~A}-\mathrm{PD}-\mathrm{C} 3-\mathrm{C} 3 \mathrm{~A}$ & $-31.0(3)$ & $\mathrm{PD}-\mathrm{C} 1-\mathrm{C} 7 \mathrm{~A}-\mathrm{C} 7$ & $118.9(8)$ \\
\hline $\mathrm{C} 2-\mathrm{C} 3-\mathrm{C} 3 \mathrm{~A}-\mathrm{C} 4$ & $170.3(7)$ & $\mathrm{C} 2-\mathrm{C} 1-\mathrm{C} 7 \mathrm{~A}-\mathrm{C} 3 \mathrm{~A}$ & $10.4(7)$ \\
\hline $\mathrm{PD}-\mathrm{C} 3-\mathrm{C} 3 \mathrm{~A}-\mathrm{C} 4$ & $-122.1(7)$ & $\mathrm{PD}-\mathrm{C} 1-\mathrm{C} 7 \mathrm{~A}-\mathrm{C} 3 \mathrm{~A}$ & $-58.8(5)$ \\
\hline $\mathrm{C} 2-\mathrm{C} 3-\mathrm{C} 3 \mathrm{~A}-\mathrm{C} 7 \mathrm{~A}$ & $-11.8(7)$ & $\mathrm{C} 2-\mathrm{C} 1-\mathrm{C} 7 \mathrm{~A}-\mathrm{PD}$ & $69.2(5)$ \\
\hline $\mathrm{PD}-\mathrm{C} 3-\mathrm{C} 3 \mathrm{~A}-\mathrm{C} 7 \mathrm{~A}$ & $55.8(5)$ & $\mathrm{C} 2-\mathrm{PD}-\mathrm{C} 7 \mathrm{~A}-\mathrm{C} 7$ & $-162.4(8)$ \\
\hline $\mathrm{C} 2-\mathrm{C} 3-\mathrm{C} 3 \mathrm{~A}-\mathrm{PD}$ & $-67.6(4)$ & $\mathrm{C} 1-\mathrm{PD}-\mathrm{C} 7 \mathrm{~A}-\mathrm{C} 7$ & $-119.3(9)$ \\
\hline $\mathrm{C} 2-\mathrm{PD}-\mathrm{C} 3 \mathrm{~A}-\mathrm{C} 4$ & $162.5(8)$ & $\mathrm{C} 3-\mathrm{PD}-\mathrm{C} 7 \mathrm{~A}-\mathrm{C} 7$ & $152.5(8)$ \\
\hline $\mathrm{C} 1-\mathrm{PD}-\mathrm{C} 3 \mathrm{~A}-\mathrm{C} 4$ & $-151.5(8)$ & $\mathrm{CL}^{\mathrm{iv}}-\mathrm{PD}-\mathrm{C} 7 \mathrm{~A}-\mathrm{C} 7$ & $-33.1(7)$ \\
\hline $\mathrm{C} 3-\mathrm{PD}-\mathrm{C} 3 \mathrm{~A}-\mathrm{C} 4$ & $119.7(8)$ & $\mathrm{CL}-\mathrm{PD}-\mathrm{C} 7 \mathrm{~A}-\mathrm{C} 7$ & $69.8(8)$ \\
\hline $\mathrm{CL}^{\mathrm{iv}}-\mathrm{PD}-\mathrm{C} 3 \mathrm{~A}-\mathrm{C} 4$ & $-77.9(7)$ & $\mathrm{C} 3 \mathrm{~A}-\mathrm{PD}-\mathrm{C} 7 \mathrm{~A}-\mathrm{C} 7$ & $119.4(9)$ \\
\hline $\mathrm{CL}-\mathrm{PD}-\mathrm{C} 3 \mathrm{~A}-\mathrm{C} 4$ & $28.5(7)$ & $\mathrm{C} 2-\mathrm{PD}-\mathrm{C} 7 \mathrm{~A}-\mathrm{C} 3 \mathrm{~A}$ & $78.2(4)$ \\
\hline $\mathrm{C} 7 \mathrm{~A}-\mathrm{PD}-\mathrm{C} 3 \mathrm{~A}-\mathrm{C} 4$ & $-115.9(8)$ & $\mathrm{C} 1-\mathrm{PD}-\mathrm{C} 7 \mathrm{~A}-\mathrm{C} 3 \mathrm{~A}$ & $121.3(6)$ \\
\hline $\mathrm{C} 2-\mathrm{PD}-\mathrm{C} 3 \mathrm{~A}-\mathrm{C} 7 \mathrm{~A}$ & $-81.5(4)$ & $\mathrm{C} 3-\mathrm{PD}-\mathrm{C} 7 \mathrm{~A}-\mathrm{C} 3 \mathrm{~A}$ & $33.1(4)$ \\
\hline $\mathrm{C} 1-\mathrm{PD}-\mathrm{C} 3 \mathrm{~A}-\mathrm{C} 7 \mathrm{~A}$ & $-35.6(4)$ & $\mathrm{CL}^{\mathrm{iv}}-\mathrm{PD}-\mathrm{C} 7 \mathrm{~A}-\mathrm{C} 3 \mathrm{~A}$ & $-152.5(3)$ \\
\hline $\mathrm{C} 3-\mathrm{PD}-\mathrm{C} 3 \mathrm{~A}-\mathrm{C} 7 \mathrm{~A}$ & $-124.4(6)$ & $\mathrm{CL}-\mathrm{PD}-\mathrm{C} 7 \mathrm{~A}-\mathrm{C} 3 \mathrm{~A}$ & $-49.6(5)$ \\
\hline $\mathrm{CL}^{\mathrm{iv}}-\mathrm{PD}-\mathrm{C} 3 \mathrm{~A}-\mathrm{C} 7 \mathrm{~A}$ & $38.0(4)$ & $\mathrm{C} 2-\mathrm{PD}-\mathrm{C} 7 \mathrm{~A}-\mathrm{C} 1$ & $-43.1(4)$ \\
\hline $\mathrm{CL}-\mathrm{PD}-\mathrm{C} 3 \mathrm{~A}-\mathrm{C} 7 \mathrm{~A}$ & $144.5(3)$ & $\mathrm{C} 3-\mathrm{PD}-\mathrm{C} 7 \mathrm{~A}-\mathrm{C} 1$ & $-88.2(5)$ \\
\hline $\mathrm{C} 2-\mathrm{PD}-\mathrm{C} 3 \mathrm{~A}-\mathrm{C} 3$ & $42.9(4)$ & $\mathrm{CL}^{\mathrm{iv}}-\mathrm{PD}-\mathrm{C} 7 \mathrm{~A}-\mathrm{C} 1$ & $86.3(4)$ \\
\hline $\mathrm{C} 1-\mathrm{PD}-\mathrm{C} 3 \mathrm{~A}-\mathrm{C} 3$ & $88.8(4)$ & $\mathrm{CL}-\mathrm{PD}-\mathrm{C} 7 \mathrm{~A}-\mathrm{C} 1$ & $-170.9(4)$ \\
\hline $\mathrm{CL}^{\mathrm{iv}}-\mathrm{PD}-\mathrm{C} 3 \mathrm{~A}-\mathrm{C} 3$ & $162.4(3)$ & $\mathrm{C} 3 \mathrm{~A}-\mathrm{PD}-\mathrm{C} 7 \mathrm{~A}-\mathrm{C} 1$ & $-121.3(6)$ \\
\hline
\end{tabular}

